\title{
The Organization and Connections of Somatosensory Cortex in Marmosets
}

\author{
Leah A. Krubitzer and Jon H. Kaas \\ Department of Psychology, Vanderbilt University, Nashville, Tennessee 37240
}

Microelectrode mapping methods were used to define and describe 3 representations of the body surface in somatosensory cortex of marmosets: S-I proper or area $3 \mathrm{~b}$ of anterior parietal cortex, S-II, and the parietal ventral area (PV) of the upper bank of the lateral sulcus. In the same animals, injections of anatomical tracers were placed into electrophysiologically determined sites in area $\mathbf{3 b}$ or S-II. Mapping results and patterns of connections were later related to architectonic fields that were delimited in sections cut parallel to the surface of manually flattened cortex and stained for myelin.

There were several major results. (1) Recordings from area $3 \mathrm{~b}$ revealed a characteristic somatotopic organization of foot to face in a mediolateral sequence as previously reported in other members of the marmoset family (Carlson et al., 1986). (2) Multiple injections of WGA-HRP in area 3b demonstrated dense, patchy interconnections with ipsilateral S-II, PV, area $3 \mathrm{a}$, and area 1 , less dense interconnections with primary motor cortex $(M-I)$, the supplementary motor area (SMA), limbic cortex of the medial wall (L), and rostrolateral parietal cortex of the lateral sulcus (PR), and callosal connections with areas 36 , S-II, and PV. Injections of 3 different tracers into the representation of 3 body regions in area $3 b$ indicated that the connections with areas $3 a, 3 b, 1,5-I I$, and PV are topographically organized. (3) Recordings from cortex on the upper bank of the lateral sulcus demonstrated a somatotopic representation of the body surface that matches that of $\mathrm{S}-11$ of other mammals. S-II immediately adjoined areas $3 \mathrm{~b}$ along the dorsal lip of the lateral sulcus. The face representation in S-II was adjacent to the face representation in $3 b$ while the trunk, hindlimb, and forelimb were represented in a caudorostral sequence deeper in the sulcus. (4) Injections in S-II revealed ipsilateral connections with areas $3 a, 3 b, 1$, a presumptive area 2, PV, PR, M-I, SMA, limbic cortex, the frontal eye fields, and the frontal ventral visual area. Dense callosal connections were with S-II and PV. (5) The recordings also revealed a systematic representation just rostral to $S-I I$ that has not been previously described in primates. The $P V$, named after its probable homolog in rodents (Krubitzer et al., 1986), roughly mirrors S-II in somatotopic organization, so that the

\footnotetext{
Received Aug. 17, 1989; revised Sept. 26, 1989; accepted Sept. 28, 1989.

We thank Sherrie Florence, Preston Garraghty, Anne Morel, and John Wall for helpful comments on this manuscript. The research was supported by NIH grant NS 16446.

Correspondence should be addressed to Jon H. Kaas, A \& S Psychology Building, Department of Psychology, Vanderbilt University, Nashville, TN 37240.

Copyright (C) 1990 Society for Neuroscience $0270-6474 / 90 / 030952-23 \$ 02.00 / 0$
}

face is represented along the area $3 b$ border and the limbs are represented deeper in the sulcus, as in S-II, but the forelimb is caudal to the hindlimb and the trunk is rostrolateral in the representation.

The results are consistent with the view that area $3 \mathrm{~b}$ is an early station in cortical processing, and area 1, S-II, and PV are higher stations. While the major cortical connections of area $3 \mathrm{~b}$ are with subdivisions of somatosensory cortex, S-II relates to motor and visuomotor fields as well. Evidence for PV in both rodents and primates suggests that this somatotopic representation, in addition to $\mathbf{S - I}(3 \mathrm{~b})$ and $\mathrm{S}-\mathrm{II}$, is common to a wide range of mammals.

Mammals appear to vary in the number of cortical somatosensory areas from a few to many (e.g., Kaas, 1987a, b), but it is uncertain how processing across cortical areas is similar or different in different types of brains. One way to approach this problem is to compare patterns of connections for those cortical subdivisions that most mammals have in common. Connection patterns help define other cortical areas within a system and suggest sequences of information flow. In the visual system, for example, area 17 or V-I (see Appendix for list of abbreviations) is widely recognized as a subdivision of visual cortex that is common to most or all mammals, and projection patterns of V-I suggest that a second area, V-II, and perhaps an additional area are basic components of the mammalian visual system (see Kaas, 1989).

In the somatosensory system, 2 cortical fields, the first (S-I) and second (S-II) somatosensory areas have been described in a wide range of mammals, but there has been some ambiguity about what is S-I. Specifically, 4 architectonic fields (areas 3a, $3 \mathrm{~b}, 1$, and 2) have been commonly included within a single field, S-I, of monkeys and other "higher" primates. However, there is now extensive evidence that each of the 4 fields contains a separate representation of the body (see Kaas and Pons, 1988, for review), and only area $3 \mathrm{~b}$ appears to be the homolog of S-I of other mammals, including prosimian primates (see Merzenich et al., 1978; Kaas, 1983). There have been similar though less notable ambiguities about the identification of S-II (see Burton, 1986). Now there is general acceptance that S-II is a single somatotopic representation of the body surface that has a characteristic "upright" homuncular orientation (see Nelson et al., 1979) and either adjoins S-I along congruent representations of the dorsal midline of the face in nonprimates (see Nelson et al., 1979; Burton et al., 1982; Clemo and Stein, 1983) and prosimians (Burton and Carlson, 1986), or along one of the representations in anterior parietal cortex (areas $3 \mathrm{a}, 3 \mathrm{~b}, 1$, and 2) of simian primates (e.g., Friedman et al., 1986; Cusick et al., 1989). 
Given the progress in resolving this problem of identification, it now seems possible to describe and compare the connections of at least 2 subdivisions of somatosensory cortex, S-I and S-II, as homologous fields across species in the major branches of mammalian evolution. Although there have been a number of descriptions of the cortical connections of S-I (e.g., rats: Akers and Killackey, 1978; Welker et al., 1988; squirrels: Krubitzer et al., 1986; cats: Jones and Powell, 1968) or area 3b (e.g., New World monkeys: Jones and Wise, 1977; Cusick et al., 1985; Old World monkeys: Jones and Wise, 1977; Vogt and Pandya, 1978) and of S-II (e.g., squirrels: Krubitzer et al., 1986; cats: Clemo and Stein, 1983; Burton and Kopf, 1984; Alloway and Burton, 1985; and Old World monkeys: Friedman et al., 1986), studies have been limited to a few mammalian species, and even in these species only a few studies have been extensive enough to provide a comprehensive description of cortical connections. Remarkably, the complete areal cortical connection patterns of area $3 \mathrm{~b}$ and of S-II have not yet been described for any primate.

Our major goal in the present investigation was to determine in detail the cortical connection patterns of area $3 \mathbf{b}$ and S-II in marmosets. Recordings were used to identify these fields for injections, and when recordings revealed a previously unknown representation in cortex rostral to S-II which we term the parietal ventral area (PV), a second goal of describing this field emerged. Marmosets were used, in part, because their small, smooth brains provide technical advantages. Their cortex can be easily separated from the rest of the brain, flattened into a single, nearly unbroken sheet, and sectioned parallel to the surface. Such sections provide direct demonstrations of areal patterns of connections without introducing artifacts associated with extensive reconstructions of frontal or sagittal sections (e.g., Krubitzer et al., 1986). Marmosets were also used because they occupy a unique position in primate evolution. Marmosets and tamarins, as members of the family Callitrichidea, have long been regarded as "the most primitive of all living monkeys" (Beattie, 1927). They appear to have changed little after evolving from stem platyrhine stock (Hershkovitz, 1977). If, as some evidence already suggests (see Carlson et al., 1986), the somatosensory cortex is less specialized in marmosets than in other monkeys, studies on marmosets could provide information that is critical for developing a theory of the evolution of the complex somatosensory system of higher primates. Part of these results have been briefly presented elsewhere (Krubitzer and Kaas, 1986).

\section{Materials and Methods}

Microelectrode multiunit recordings were used to identify and characterize subdivisions of somatosensory cortex in 9 adult marmoset monkeys (Callithrix jacchus). In 6 of the same animals, patterns of connections were demonstrated by injecting anatomical tracers into area $3 \mathrm{~b}$, S-II, or both cortical fields. The physiological and anatomical results were subsequently related to cortical architecture in brain sections cut parallel to the cortical surface in 5 cases and in one case cut coronally. The recording techniques and anatomical procedures closely followed those used previously (Krubitzer and Kaas, 1986; Luethke et al., 1989).

Surgery. Sterile surgical procedures were followed for all combined recording and injection experiments. Each animal was anesthetized with ketamine hydrochloride, $30 \mathrm{mg} / \mathrm{kg}$, IM (White et al., 1982) and acepromazine, $1 \mathrm{mg} / \mathrm{kg}$, IM. Both were supplemented as needed to maintain a surgical level of anesthesia. In addition, a local anesthetic, 2\% xylocaine hydrochloride, was injected subcutaneously at the scalp and where ear bars entered the ear canals. Part of somatosensory cortex was exposed and protected in an acrylic chamber filled with silicone fluid. A photograph of the exposed brain surface was used to guide the placement of electrode recording sites. After recordings and injections were com- plete, the chamber was removed, the dura was sutured in place, the craniotomy was closed with dental acrylic, and the skin sutured. The animals were carefully monitored during recovery from anesthesia and maintained for survival periods of $2-3 \mathrm{~d}$.

Recordings. Low-impedance tungsten microelectrodes $(0.95-1.5 \mathrm{M} \Omega$ at $1000 \mathrm{~Hz}$ ) were used to record from clusters of neurons in the middle layers of cortex. The electrode was advanced with a stepping microdrive, and recording depths were noted. Electrode penetrations were perpendicular to the cortical surface in area $3 \mathrm{~b}$. Because $\mathrm{S}-\mathrm{II}$ and $\mathrm{PV}$ are located on the upper bank of the lateral sulcus, recordings were obtained by directing the electrode parallel to the cortical layers. Sulci penetrations parallel to the cortical layers extended $3-5 \mathrm{~mm}$, and recordings were made every $200-400 \mu \mathrm{m}$ along these penetrations. Receptive fields for neurons were determined by stimulating the skin with light taps or small probes and by displacing hairs. Responses to pressure and moving body parts were also noted. Small electrolytic lesions $(10 \mu \mathrm{A}$ for $6 \mathrm{sec})$ were placed at physiological boundaries and other sites of interest for later correlation of anatomical and physiological results.

Injections and histology. Calibrated micropipettes were used to inject anatomical tracers into selected sites in area $3 \mathrm{~b}$ and S-II that were identified by the recordings. In 2 animals, $85-29$ and 85-26, mediolateral rows of up to 12 separate injections were centered in area $3 \mathrm{~b}$. Each injection consisted of $0.05-0.1 \mu \mathrm{l}$ of $0.1 \%$ wheatgerm agglutinin conjugated to horseradish peroxidase (WGA-HRP). In addition, in an attempt to distinguish connections from different body representations of $3 \mathrm{~b}$ in the same animal, marmoset $85-51$ had 3 different tracers injected into different parts of area $3 \mathrm{~b}$. Two closely spaced injections of $3 \%$ Fast blue $(0.2-0.4 \mu \mathrm{l})$ were placed medially in the portion of area $3 \mathrm{~b}$ representing the hindlimb, 2 closely spaced injections of WGA-HRP $(0.05$ $\mu 1)$ were placed in the forelimb representation, and 2 closely spaced injections of $4 \%$ fluoro-gold $(0.3-0.5)$ were placed in the face representation. In 3 marmosets, WGA-HRP $(0.05-0.1 \mu \mathrm{l})$ was injected into the forelimb region of S-II. In one of these animals (86-58), additional injections of Fast blue and diamadino yellow were placed in the hand and wrist representations of area $3 \mathrm{~b}$.

After survival periods of $48 \mathrm{hr}$ for WGA-HRP and $72 \mathrm{hr}$ for fluorescent dyes, the animals were given a lethal dose of sodium thiopental and transcardially perfused with $0.9 \%$ saline followed by $2 \%$ paraformaldehyde in phosphate butter and $2 \%$ paraformaldehyde in $10 \%$ sucrose phosphate buffer. After the perfusion, the brain was removed, and the cortex was peeled from the brain stem and thalamus. The lateral sulcus was opened, the cortex was manually flattened between glass slides, and the flattened cortex was soaked overnight in $30 \%$ sugar phosphate buffer. All cortices but one were cut parallel to the cortical surface on a freezing microtome into $40 \mu \mathrm{m}$ sections. One cortical hemisphere was cut coronally into $50 \mu \mathrm{m}$ sections. Alternate sections were reacted for HRP with tetramethylbenzidine (TMB; Mesulam, 1978) or stained for myelin using the Gallayas (1979) silver procedure. For cases where fluorescent dyes were injected, 1 in every 3 sections were reacted for HRP, stained for myelin, or mounted for fluorescent microscopy.

Data analysis. Enlarged drawings of brain sections were used to plot labeled neurons and axon terminals from every section reacted for HRP or mounted for fluorescent microscopy. In addition, lesions placed at physiological boundaries, blood vessels, and tissue artifacts were also added to these reconstructions. Adjacent sections stained for myelin were drawn at the same magnification, and architectonic boundaries, blood vessels, and lesions were marked on these sections. By matching blood vessels, lesions, and tissue artifacts, architectonic boundaries could be added to sections with tracers. Recording sites in and around area $3 \mathrm{~b}$ and $\mathrm{S}-\mathrm{II}$ were related to these surface-view reconstructions by using marker lesions to identify some of the recording sites. The locations of other recording sites were determined by measuring distances from marker lesions and other landmarks. For recording sites in cortex of the lateral sulcus, it was usually possible to identify complete electrode tracts as well as marker lesions from a single section.

\section{Results}

In the present experiments, microelectrode recordings were used to identify or define subdivisions of somatosensory cortex for injections of WGA-HRP or fluorescent dycs. Later, both electrophysiological and anatomical results were related to myeloarchitecture in brain sections cut parallel to the surface of manually flattened cortex. Results are presented in 3 parts. First, 
Figure 1. Dorsolateral view of marmoset cortex showing the locations of area $3 b$ in anterior parietal cortex and S-II and the parietal ventral area $(P V)$ in cortex of the lateral sulcus (black). Other somatosensory, motor and visual areas are in thin black lines. $M$, motor cortex; $F E F$, frontal eye field; $F V$, frontal ventral visual area; $S M A$, supplementary motor area; $M T$, middle temporal visual area; $l s$, lateral sulcus.

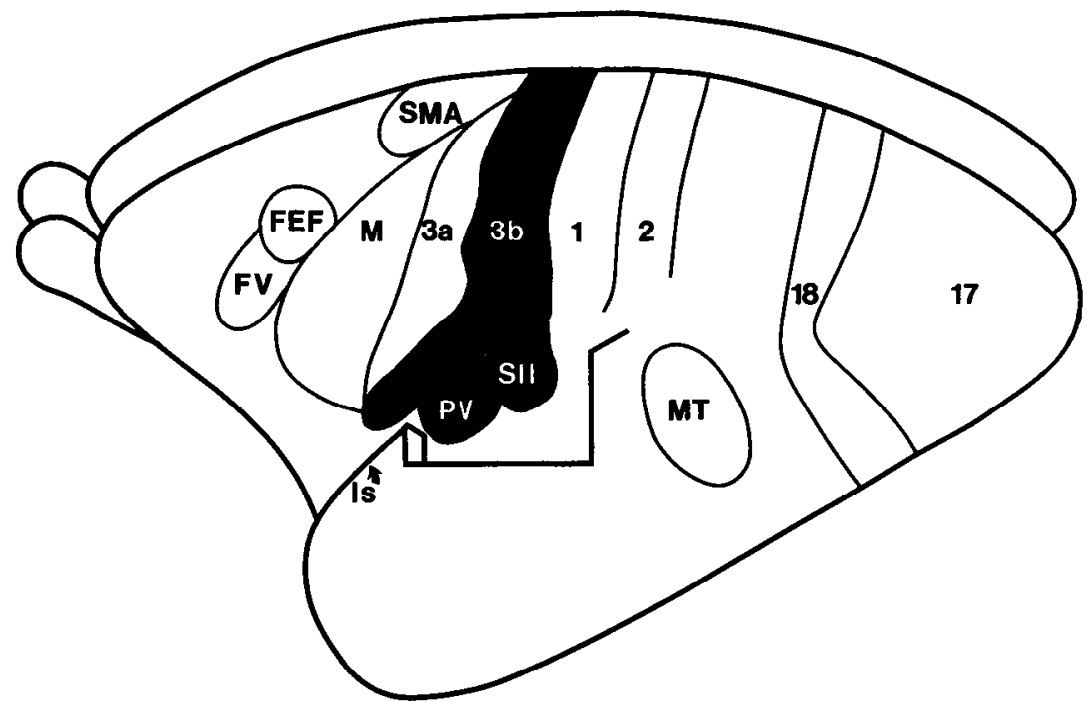

we present evidence from microelectrode recordings for subdividing anterior parietal and lateral parietal cortex into a number of functionally distinct fields. Second, we describe architectonic fields that are distinguishable in sections stained for myelin and show that some of these fields correspond to electrophysiological maps. Finally, we describe areal patterns of ipsi- and contralateral cortical connections resulting from the injection of tracers in areas $3 \mathrm{~b}$ and S-II. The proposed subdivisions of somatosensory cortex are shown in Figure 1.

\section{Microelectrode maps}

Anterior parietal cortex

The somatotopic organization of anterior parietal cortex was most extensively explored in 3 cases where multiple injections of tracers were placed within S-I proper or area $3 \mathrm{~b}$ (Fig. 2, $A$, $B)$. In each of these cases, recordings revealed a systematic representation of cutaneous receptors in the S-I region. Receptive fields for the most medial recording sites were on the foot, while the leg, trunk, arm, hand, face, and mouth were represented in successively more lateral locations. As described by Carlson et al. (1986), the digits of the hand were represented from $1-5$ in a lateromedial sequence in the rostral half of S-I, with the tips of the digits activating the rostral border. The palm activated cortex caudal to the digits. More laterally, skin locations along the dorsal midline of the head, including regions near the ear, eye, nose, and upper lip activated the caudal border of S-I, in a mediolateral sequence, while the lower lip and teeth related to the rostral border. Receptive fields for neurons in $3 \mathrm{~b}$ varied in size depending on the body part as previously described (Carlson et al., 1986) and were smaller than those in PV and S-II (Figs. 3, 4).

Most of the cortex surrounding S-I was much less responsive to cutaneous stimuli. Immediately rostral to S-I, in cortex that was later identified architectonically as area $3 \mathrm{a}$, neurons were typically unresponsive to cutaneous stimuli, although responses were sometimes elicited by stimulating deep peripheral receptors using moderate pressure and manipulation of body parts. Occasionally, neurons could be activated by less intense stimuli, especially on the claws and digits of the hand. Recording sites whose neurons were activated by stimulating the foot were me- dial to those activated by stimulating the hand (Fig. 2, $A, B$ ). Sites in area 1 caudal to $\mathrm{S}-\mathrm{I}$ were much less responsive to cutaneous stimulation than those in S-I. Usually, neurons in area 1 were unresponsive to tactile stimuli, but light taps to the skin of the hand sometimes produced responses in cortex caudal to the representation of the hand in S-I.

Cortex lateral to S-I, on the upper bank of the lateral sulcus, had 2 additional representations of the body surface, S-II and PV, that were activated by light cutaneous stimulation of peripheral receptors. The more caudal of these representations was identified as S-II on the basis of relative position and somatotopic similarities with the region termed S-II in other mammals (see Discussion). The more rostral representation resembled PV of squirrels (Krubitzer et al., 1986) in somatotopic organization and relative position and was termed PV. These representations were distinguished from S-I by changes in somatotopic patterns and differences in receptive field sizes.

\section{The second somatosensory area, $S-I I$}

Cortex just lateral to S-I was explored in 5 marmosets by extending microelectrode penetrations along the middle layers of cortex on the upper bank of the lateral sulcus. S-II was 1 of 2 systematic representations of cutaneous receptors that were identified. S-II adjoins the caudolateral portion of S-I representing the upper lip. Although the portion of S-II adjacent to S-I also represents portions of the rostral face including the upper lip, recording sites were distinguished from those in S-I by several features. First, when recording sites crossed the S-I/ S-II border, a reversal of the somatotopic pattern was apparent. Receptive fields for neurons in rows of recording sites from S-I to S-II progressed from the lateral to the medial upper lip in S-I, and from the medial upper lip to the lateral and caudal face in S-II (Fig. 4, penetrations $a-d$ ). Second, receptive fields were much larger in S-II than S-I. Finally, although neurons in S-II were highly responsive to cutaneous stimuli, they were often somewhat less responsive than neurons in S-I.

Caudally in the border region of S-I and S-II, sites were first activated by the nose and the upper rostral face and then by the upper lateral face between the eye and the ear for sites deeper in the sulcus (see Fig. 3, sites 1-3; Fig. 4, b-d). In more rostral 
cortex, sites had receptive fields that included more of the lower face, although the progression again was from rostral to caudal face with deeper recording sites (Figs. 5-7).

Other body regions were represented in parts of S-II deeper in the sulcus. Caudally, successively deeper recording sites resulted in receptive field progressions from the upper face and head to the back of the neck and upper trunk, and the lower trunk and tail (Fig. 3, sites 1-8). The orientation of S-II varied across individuals, so the sites related to the lower trunk and tail were caudomedial in some animals (Figs. 5, 7) and more lateral in others (Fig. 6).

Locations rostral to the representation of the trunk, in lateral S-II, represented the leg and foot. More medial parts of S-II were devoted to the arm and hand. The representation of the glabrous digits occupied much of the rostral border of S-II. Receptive fields on the hand for sites in S-II were typically much larger than those found in S-I, although occasionally a small receptive field restricted to a portion of a single digit was noted in S-II (Fig. 5, field 5). Because most receptive fields on the hand typically included several or all digits, a strict topographic organization for the digits was not observed. However, sites closer to S-I were better activated by digit 1 , while sites deeper in the sulcus tended to be better activated by the lateral digits. The results indicate that S-II is roughly a mirror reversal of S-I along adjoining representations of the midline of the upper face.

All parts of S-II were responsive to light stimulation of the body surface or to movement of body hairs. Sites caudal to S-II were unresponsive or poorly responsive to cutaneous stimuli. Few recordings were obtained from cortex "lateral" (in flattened cortex) to S-II since this cortex was at the base of the lateral sulcus or on the lower bank of the lateral sulcus. Cortex in this region in owl monkeys responds to cutaneous stimuli and forms another representation of the body surface (Cusick et al., 1989).

\section{The parietal ventral area, $P V$}

Recordings in cortex rostral to S-II revealed an adjoining representation of cutaneous receptors in a somatotopic pattern that roughly mirrors that of S-II. Thus, PV and S-II have adjoining representations of the hand and face, while the foot, trunk, and proximal limbs are more distantly represented in the 2 fields. Because of the adjoining hand and face representations, the relatively large receptive fields for both areas, and a similar responsiveness to cutaneous stimuli, the exact border of PV with S-II is difficult to determine without examination of receptive field progressions for closely spaced recording sites.

Electrophysiological evidence for $\mathrm{PV}$ is presented in Figures 4-7. A reversal of a receptive field progression for rows of sites extending from S-II into PV marks the border region between these 2 fields. In Figure 4, for example, sites 1-5 are largely in a single electrode penetration that was favorably angled to cross the S-II/PV border. A receptive field progression extended from the dorsal forearm and wrist onto the hairy digits, remained on the hairy digits, and then reversed onto the wrist and the forearm. A reversal of the somatotopic progression occurred between sites 3 and 4 . At a grosser level, a row of recording sites in separate electrode penetrations (Fig. 6, row 1) shows a progression of receptive fields from the upper trunk in caudal S-II, down the forearm, to the digits of the hand in rostral S-II, back up the hand and forearm in caudal PV, and finally to the upper trunk midline again in rostral PV (also see receptive fields for sites $1-7$ in Fig. 5).

Enough recording sites were studied within PV in each of 3
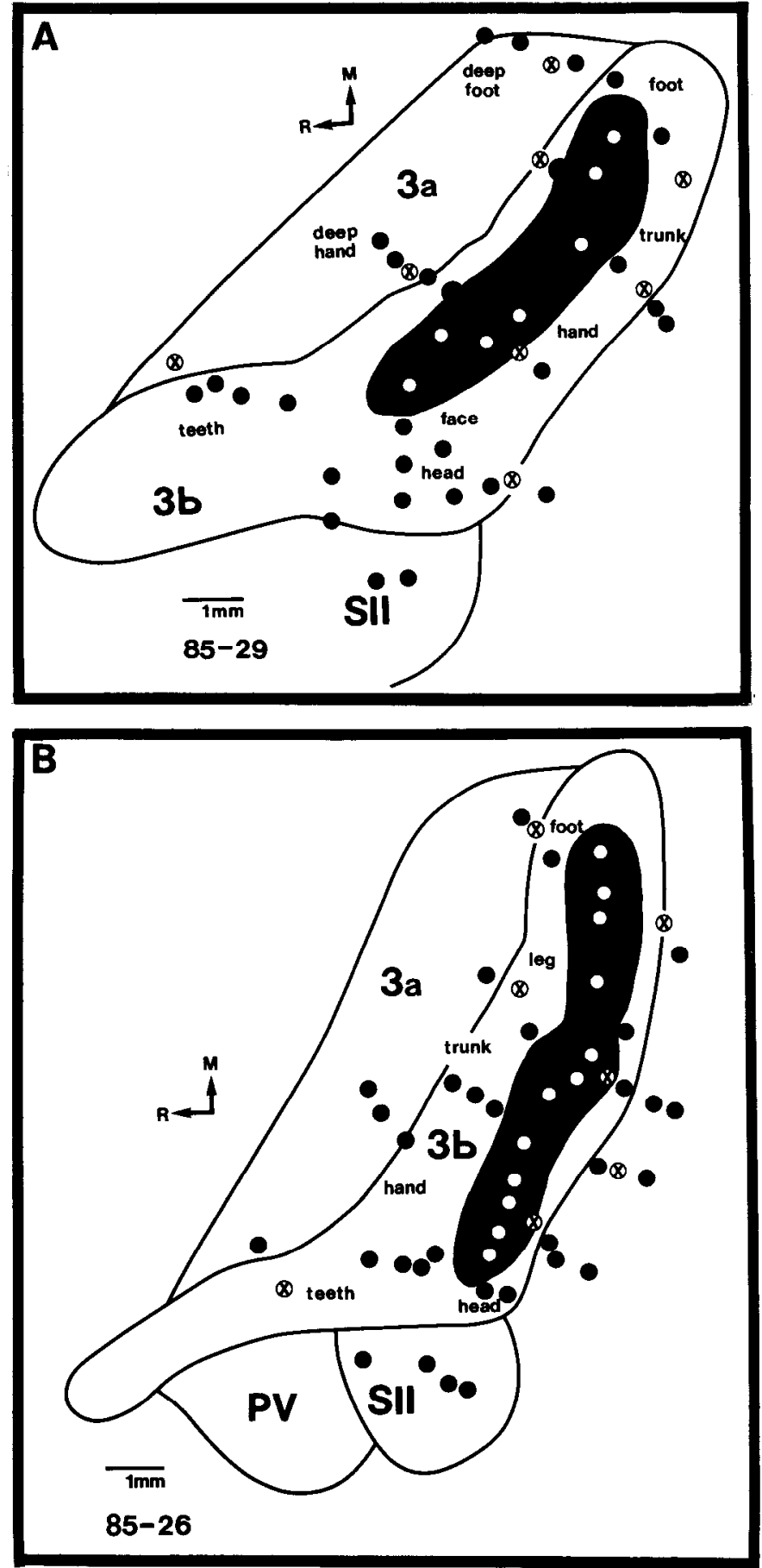

Figure 2. Partial map of area $3 \mathrm{~b}$ and the location of a strip of WGAHRP injections relative to that map for marmoset 85-29 $(A)$ and 85$26(B)$. In both cases, the foot is represented most medially in $3 \mathrm{~b}$; followed progressively by the trunk, hand, and face representations in more lateral cortex. Responses to stimulating muscles and joints were obtained for neurons in area 3a. Filled black circles mark electrode penetrations with neurons responsive to somatic stimuli; open circles with $\times$ 's, lesions placed at physiological boundaries of area $3 \mathrm{~b}$; open white circles, punctures of the micropipette for injections. The uptake area of the injection sites is in solid black. Lines denote architectonic boundaries. 
Figure 3. Location of an injection of WGA-HRP relative to a partial map of S-II in marmoset 86-55 (upper right). The location of S-II relative to $3 \mathrm{~b}$ and the lateral sulcus are depicted on a dorsolateral view of the brain on the upper left. Receptive fields of neurons for numbered and lettered recording sites in S-II are illustrated in the lower half of this figure. Receptive fields move from head to tail as recording sites in S-II progress away from the S-I border. Also, as recording sites cross the $3 \mathrm{~b} / \mathrm{S}$ II border, receptive fields dramatically increase in size. The injection (solid black circle) in this case was placed in the representation of the arm and shoulder and included limited portions of the face and trunk representations as well. Scale bar is for the enlarged S-II area in the upper right. sh, shoulder; $t r$, trunk. Conventions as in previous figures.

Figure 4. Location of an injection of WGA-HRP relative to a partial map of $\mathrm{S}-\mathrm{II}$ in marmoset 86-58 (upper right). The locations of S-II and area $3 \mathrm{~b}$ are shown on the brain surface in upper left. Receptive fields of neurons for numbered and lettered recording sites in S-II are illustrated in the lower half of this figure. Some recording sites (4-5) are within PV, and a reversal in the progression of receptive fields is demonstrated at the S-II/PV border. The injection in this case was centered in the representation of the forearm, but it also includes parts of the representations of the face and hindlimb. The scale at lower left is for the enlarged S-II area in the upper right. $h l$, hindlimb; fa, forearm. Conventions as in previous figures.
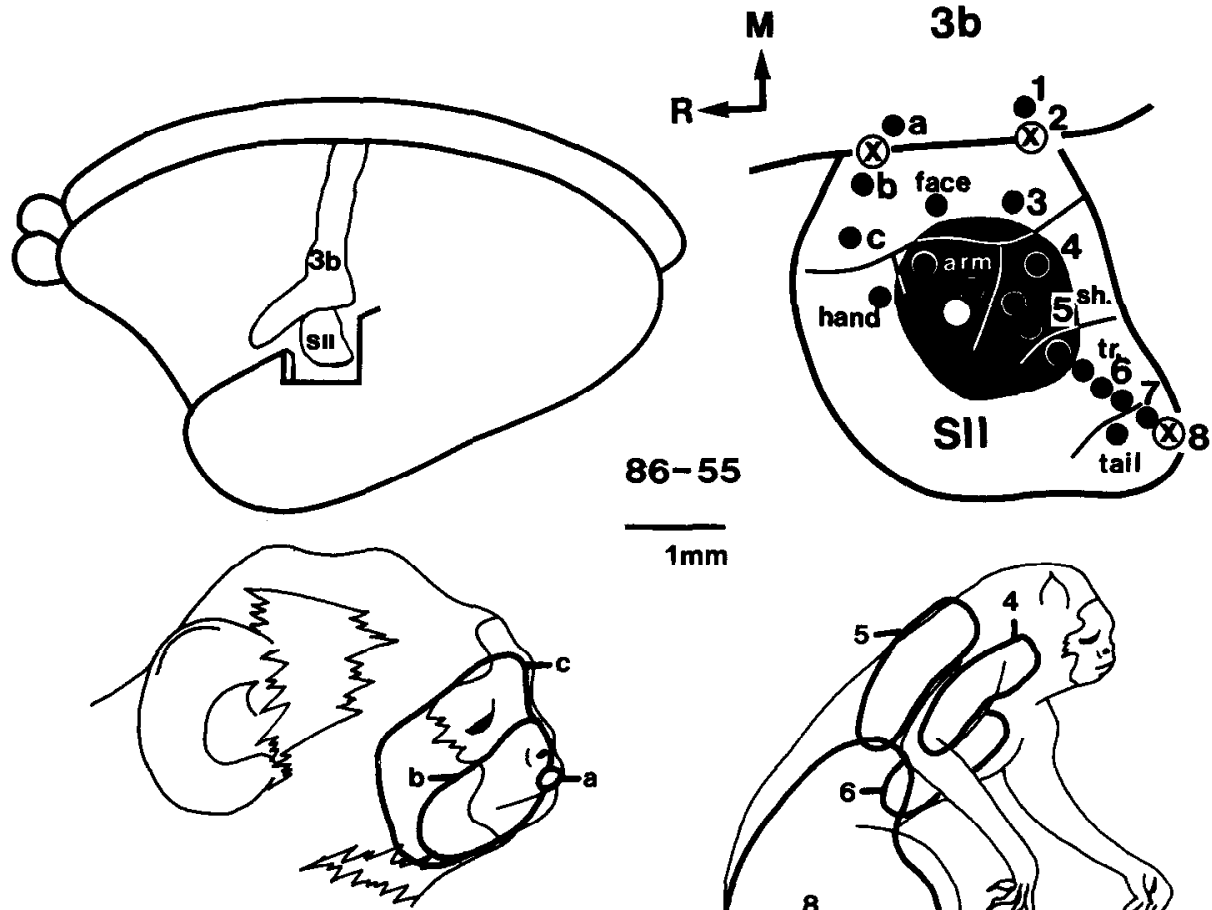

$1 \mathrm{~mm}$
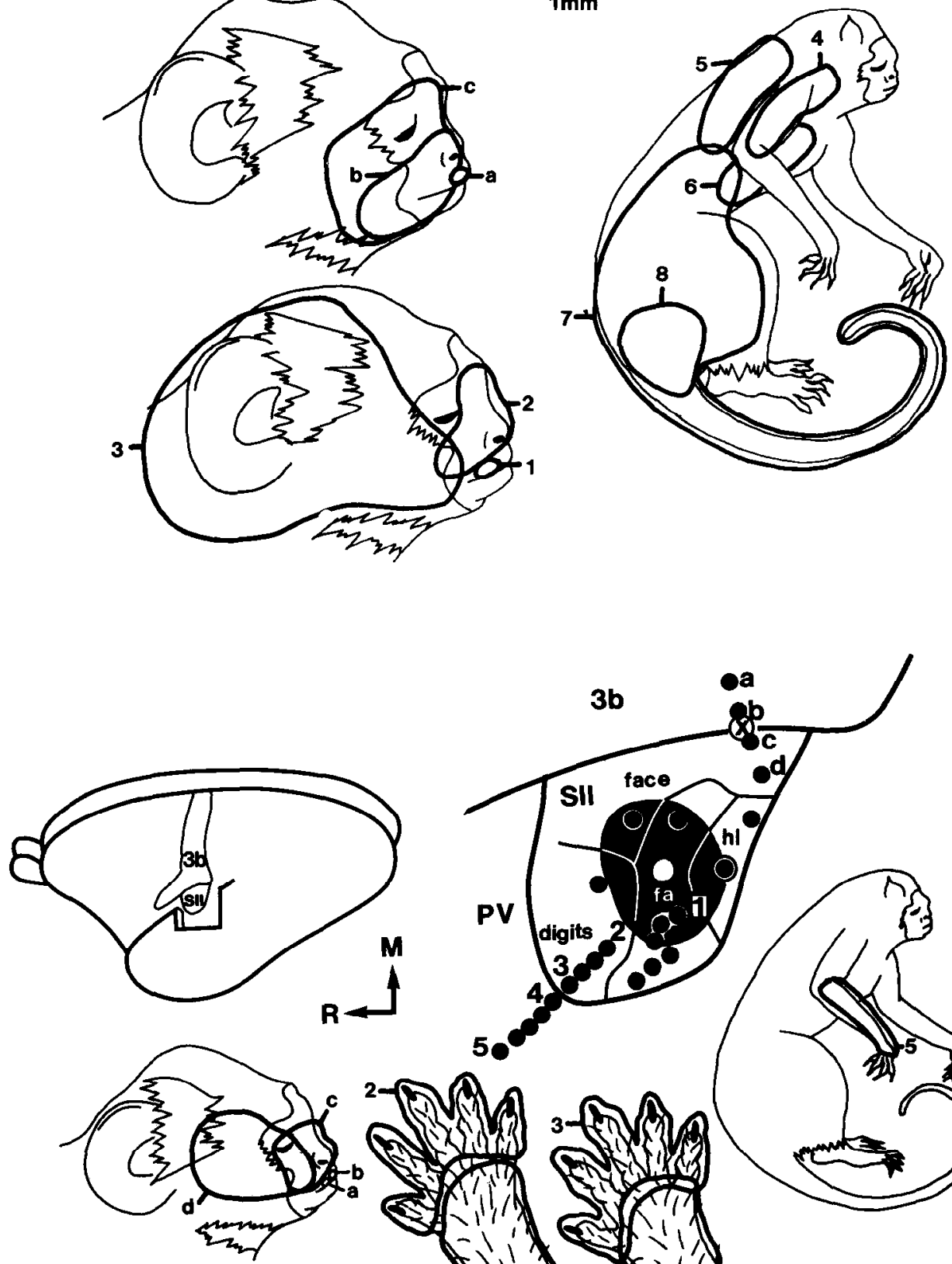

86- 58

$1 \mathrm{~mm}$

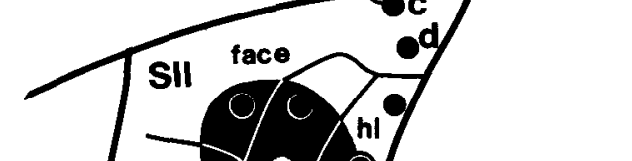

PV
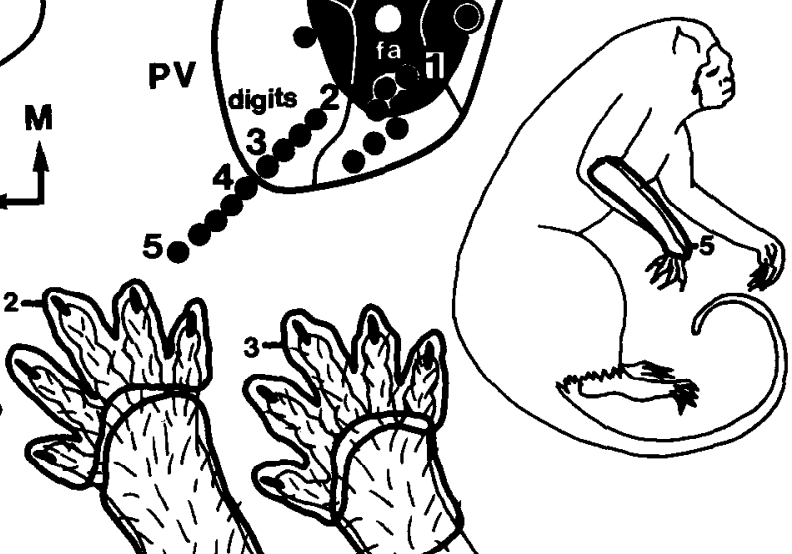
different cases to indicate individual variability within an overall somatotopic pattern (Figs. 5-7). Cortex deep to PV was not adequately sampled, so that this border region of PV was not always identified, and cortex rostral to PV was unresponsive to light cutaneous stimulation.

\section{Myeloarchitecture}

We examined parietal and frontal cortex of marmosets in both standard planes of section and in sections cut parallel to the surface of flattened cortex. Stains for cell bodies or myelin, and reactions for cytochrome oxidase all reveal subdivisions of somatosensory and motor cortex (see Carlson et al., 1986, and Krubitzer and Kaas, 1988, for previous descriptions). However, for flattened cortex, myelin stains were the most useful.

Previously, S-I of marmosets has been shown to be coextensive with cytoarchitectonic field, area 3b (Carlson et al., 1986; Krubitzer and Kaas, 1988). As in other primates, area $3 \mathrm{~b}$ is characterized by a dense packing of cells in layers IV and, to a lesser extent, in layer VI, so these layers are markedly darker in Nissl preparations in area $3 \mathrm{~b}$ than in adjoining fields. These same layers also demonstrate a high level of cytochrome oxidase (CO) activity, so that the field is conspicuous in CO preparations in both transverse and tangentially sectioned cortex. As is typical of primary sensory fields, area $3 \mathrm{~b}$ is densely myelinated. The myelin is most dense in deeper layer III and again in layers V and VI. In flattened cortex, area $3 \mathrm{~b}$ is notable as a mediolateral strip of dense myclination with a marked rostralward curvature laterally (Fig. 8, $A, B, D$ ). The curvature occurs where the rostral extension of S-I devoted to the oral cavity joins the mediolateral extension of the lips and the rest of the body. A wider region sometimes containing a less myelinated central portion is devoted to the hand. Often, an intrusion of less myelinated cortex lateral to this wider region marks the junction between the hand and face representation (Fig. $8, A, B, D$ ). Small, unmyelinated zones course through more heavily myelinated portions of the face and hindlimb as well (Fig. 8, $A, B, D$ ).

We identify a strip of cortex immediately caudal to area $3 b$ as area 1 (Krubitzer and Kaas, 1988) because it is in the relative position of area 1 and has architectonic features of area 1 of other monkeys. Area 1 in marmosets forms a $2-\mathrm{mm}$-wide band of cortex along the caudal border of area $3 \mathrm{~b}$. In Nissl preparations, both layers IV and VI are less densely packed with neurons than area $3 \mathrm{~b}$ rostrally and adjoining cortex caudally, and these layers are less reactive for $\mathrm{CO}$ than in area $3 \mathrm{~b}$. In myelin preparations from flattened cortex, area 1 is much less myelinated than area $3 \mathrm{~b}$ (Fig. $8, A, D$ ), but area 1 is slightly more myelinated than cortex caudal to area 1 . Thus, in favorable sections, a caudal border of area 1 can be identified (Fig. 8, $A, D$ ).

We refer to the cortex caudal to area 1 as area 2 because it is in the position of area 2 of other monkeys. Architectonically, this cortex is difficult to distinguish from other fields in the same region, and we have only limited evidence from connections (Figs. 15 and 17; see Discussion) that cortex in the position of area 2 is indeed area 2.

In Nissl-stained sections cut in the parasagittal plane (see figure 9 of Carlson et al., 1986, and figure 5 of Krubitzer and Kaas, 1988), a band of cortex on the rostral border of area $3 b$ of marmosets is identified as area $3 \mathrm{a}$ by a clear continuation of a granular layer IV, but a marked reduction of cell packing in both layers IV and VI and often larger pyramidal cells in layer $\mathrm{V}$. In myelin-stained sections from flattened cortex, area $3 \mathrm{a}$ is much less densely myelinated than area $3 b$, and it often appears

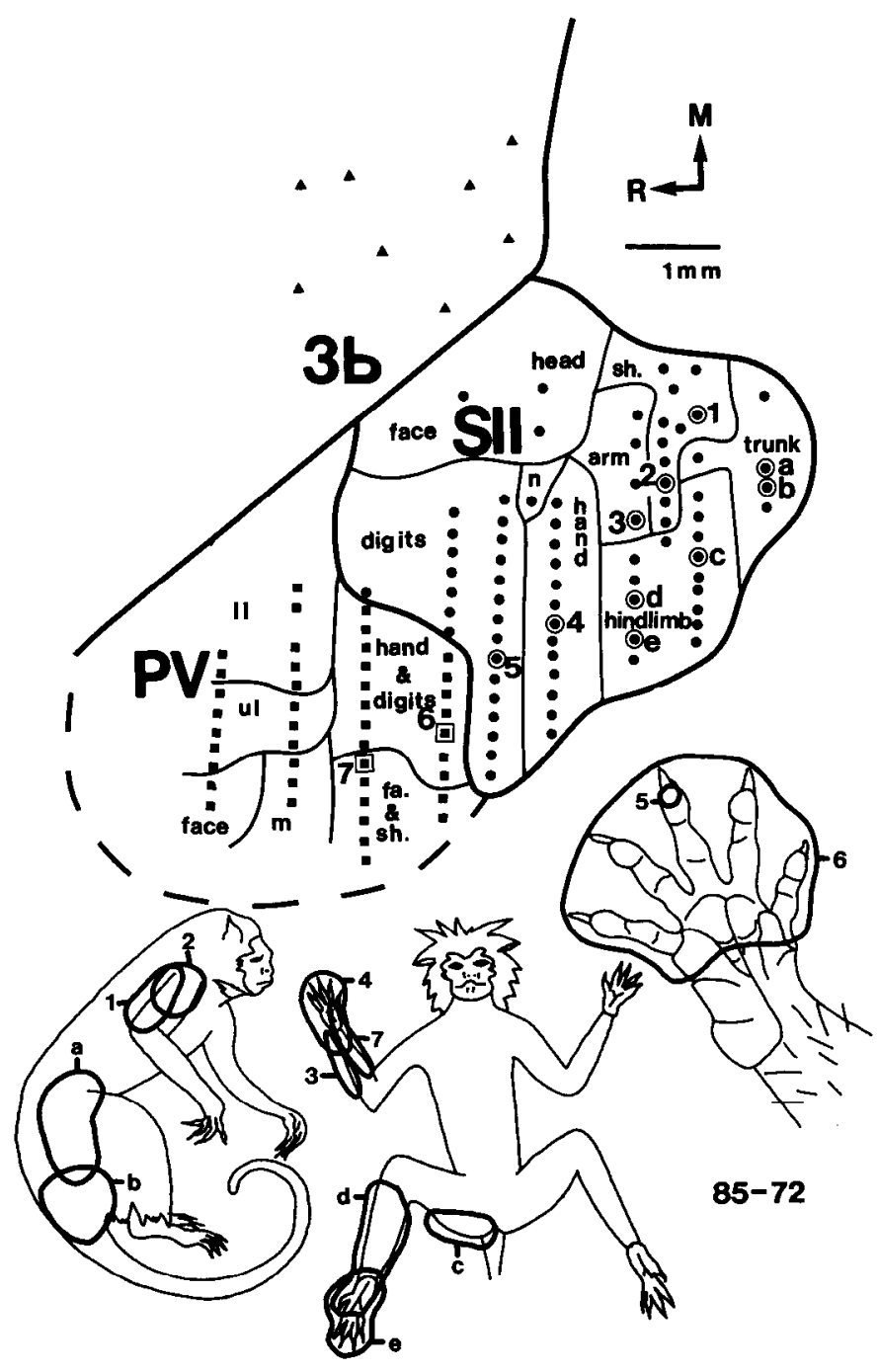

Figure 5. Somatotopic maps of S-II and PV for marmoset 85-72. A progression of recording sites from caudomedial S-II to rostrolateral PV result in a sequence of receptive fields from shoulder, forelimb, hand, and digits in S-II to digits, hand, and forelimb in PV. Thus, a reversal in the receptive field sequence identifies the S-II/PV border. Symbols: triangles, recording sites in area $3 \mathrm{~b}$; circles, in S-II; squares, in PV; $l l$, lower lip; $u l$, upper lip; $m$, mouth; $f a$, forearm; $s$, shoulder; $n$, nose. Solid lines mark architectonic boundaries; dashed lines indicate estimated boundaries. Other conventions as in previous figures.

to be slightly more myelinated than agranular motor cortex (Fig. $8, A, D)$. Thus, a rostral border of area $3 \mathrm{a}$ is often apparent, especially when a sequence of sections is studied. Further rostrally, a rostral border of primary motor cortex, M-I, can sometimes be identified by a reduction in myelin density (Fig. $8 \mathrm{~A}$ ). Medial to M-I and rostral to area $3 \mathrm{a}$, an oval of moderately dense myelination is notable in some preparations (Fig. $8 \mathrm{~A}$ ). We identify this cortex as the supplementary motor area, SMA, by position (see Gould et al., 1986), although we have no direct evidence from microelectrode stimulation experiments. Immediately rostral to M-I, small ovals of moderately dense myelination mark the frontal eye field (FEF) and the frontal visual area (FV; Fig. 8A). Previously, we identified these fields using microstimulation to evoke eye movements and by connections with visual cortex (Kaas and Krubitzer, 1988).

Cortex lateral to area $3 \mathrm{~b}$ in the region of S-II and PV differs 
Figure 6. Somatotopic maps of S-II and PV. for marmoset 89-42. A progression of recording sites from caudal S-II (row 1, $A-D)$ to rostral PV (row 1, $E-H)$ demonstrates receptive fields that progress from shoulder to digit tip in S-II and digits to shoulder in PV. Thus, a reversal in receptive fields is observed at the S-II/PV border. Recording sites in row 2 depict receptive field progressions on the hindlimb of S-II. Recording sites in row 3 show receptive field progressions on the forelimb and neck. $h$, head; dig., digits; $t r$, trunk; sh, shoulder; gen., genitals. Other conventions as in previous figures.
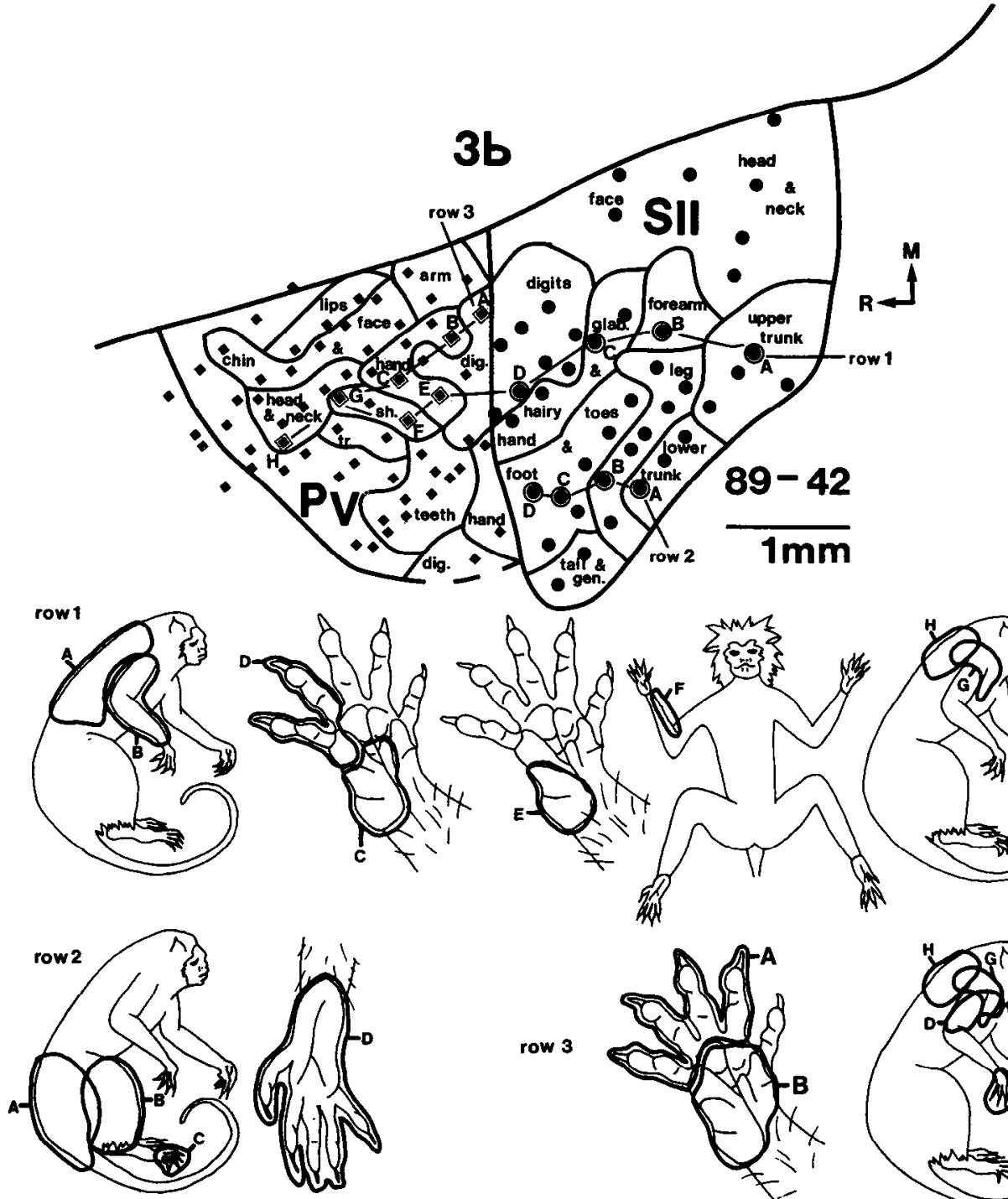

row 3
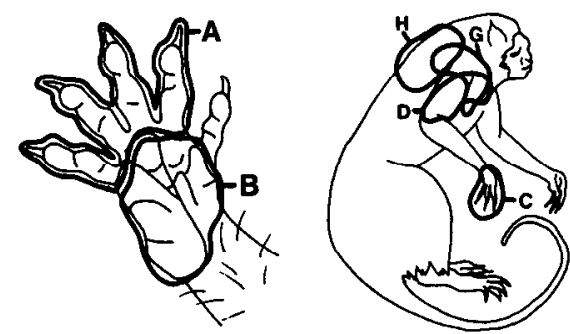

from area $3 \mathrm{~b}$ by less dense packing of neurons in layers IV and VI, although the cortex clearly has a well-developed layer IV. In cortex that has been flattened, PV and S-II can be distinguished in favorable preparations as 2 adjoining ovals of moderate myelination (Fig. 8, $A, C$ ).

Several other fields in occipital and temporal cortex are readily identified in the myelin-stained sections and serve as useful landmarks. These include the densely myelinated primary (A-I) and rostral $(R)$ auditory fields, the middle temporal visual area (MT), the fundal superior temporal area (FST), and areas 17 and 18 (Luethke et al., 1989).

\section{Cortical connections}

Connections of area $3 b(S-I)$

We investigated the interconnections of area $3 \mathrm{~b}$ with other cortical fields in 2 types of experiments. Both types of experiments were designed to determine the extent and organization of somatosensory fields, especially areas $3 \mathrm{a}$ and 1 , that are generally not responsive to cutaneous stimulation under typical recording conditions. First, we were interested in establishing the total extent of other fields that have connections with area $3 \mathrm{~b}$. We attempted this by placing a number of closely spaced injections in area $3 \mathrm{~b}$ in order to fill much of the entire area with WGAHRP but still limit the uptake zone to the boundaries of $3 \mathrm{~b}$. This was accomplished by first using microelectrode recordings to establish the rostral and caudal boundaries of S-I. In practice, to limit the recording time, we determined the boundaries of thc mediolateral extent of S-I representing the face, hand, trunk, and foot and not the rostrocaudal extension representing the oral cavity. As many as 12 injections of WGA-HRP were placed in a mediolateral row within area $3 \mathrm{~b}$. We reasoned that if we injected an entire area (3b), transported tracer would fill much of the projection zones such as $3 \mathrm{a}$ and 1 , and thus help define the extent of these fields. Second, we wanted to investigate the possibility of lopographic organization within the projection zones of area $3 \mathrm{~b}$. This was determined by injecting 3 different tracers in 3 different electrophysiologically determined locations in area $3 b$.

Multiple injections were placed in rows that formed a mediolateral strip in area $3 b$ in 3 marmosets. In each case, the injection strip extended from the representation of the foot to the head. The dense injection label was completely confined to 
area $3 b$ in 2 cases, and nearly completely in the third case. Connection patterns are illustrated for the 2 cases with completely confined injections. No notable differences were noted in the projection targets for these cases (see Fig. 2, $A, B$, for injection sites relative to the microelectrode mapping data). In brief, most of the interconnections of area $3 b$ were with 4 adjoining somatosensory fields. Label was found scattered over much of area 3a, area 1, S-II, and PV (Figs. 9, 10, 13). Although the label extended across entire fields, it was unevenly distributed in patches in all 4 fields. These results suggest either that there was a very uneven uptake and transport of label or that the injected field, area $3 \mathrm{~b}$, connects only to subsets of locations in other fields. Less label was noted in area 1 than in the other 3 fields. In case 85-29 (Fig. 9), most of the label was in the rostral half of area 1 , while some label extended nearly to the caudal border in case 85-26 (Fig. 13).

Injections in area $3 \mathrm{~b}$ produced limited amounts of label in motor cortex (M-I), the supplementary motor area (SMA), limbic cortex (L) medial to SMA, and in cortex rostral to PV in a general region of rostroventral parietal cortex we term PR for convenience. A few labeled cells were found in the general location of area 2 of case 85-26 and 85-51 (Figs. 13, 14A), and in both cases, label in the location of area 2 was only in middle and more medial portions. Thus, area 2 may project to only limited parts of area $3 \mathrm{~b}$.

A somatotopic pattern of connections of area $3 \mathrm{~b}$ with some of these fields was suggested by the locations of neurons labeled by injections of 3 different anatomical tracers in case $85-51$ (Figs. $14 A, 15$ ). Injections placed in the hindlimb/trunk, digit, and face portion of area $3 \mathrm{~b}$ formed a mediolateral sequence of label in area 1 comparable to the injection sequence in area $3 b$ (Fig. 15), suggesting that area 1 contains a representation, in parallel to that in area $3 \mathrm{~b}$, as in other monkeys.

Labeled neurons from 3 injection sites formed a more complex pattern in area 3a. Although much transported label found in area $3 \mathrm{a}$ formed a mediolateral sequence that matched that of the injection sites (Fig. 15), there was some mixture of neurons projecting to hand cortex with those projecting to hindlimb/ trunk (Fig. 14A). Finally, a lateral group of neurons projecting to hand cortex was clearly out of topographic sequence (Fig. 14). Since this label was near the lateral border of area $3 a$, one possibility is that this label is not in $3 \mathrm{a}$, but in another field.

The patterns of labeled neurons from the 3 injections were complex in areas PV and S-II also, but roughly corresponded to somatotopic sequences predicted from the mapping data. Thus, the face injection in $3 \mathrm{~b}$ labeled adjacent parts of PV and S-II that were found to represent the face (Figs. 5-7). Furthermore, hindlimb/trunk injections labeled caudolateral portions of S-II and rostral and, to a lesser extent, caudal locations in PV (Fig. 15). Label in S-II from the hand injections in $3 \mathrm{~b}$ was rostral to label from the trunk injections, and the reverse was observed in PV, as would be expected from the roughly mirrorimage organization of these 2 fields.

The label pattern also suggests that a representation exists in area 2 in parallel to those in area $3 b$ and area 1 . The few neurons in area 2 labeled by the hindlimb/trunk injection in area $3 b$ were medial to the few labeled by the hand injection. Possibly, an area 2 representation extends laterally to near the caudal margin of S-II. In that case, the few neurons caudal to S-II that were labeled by the face injection would complete the somatotopic sequence in area 2.

The locations of labeled cells in motor cortex and SMA suggest

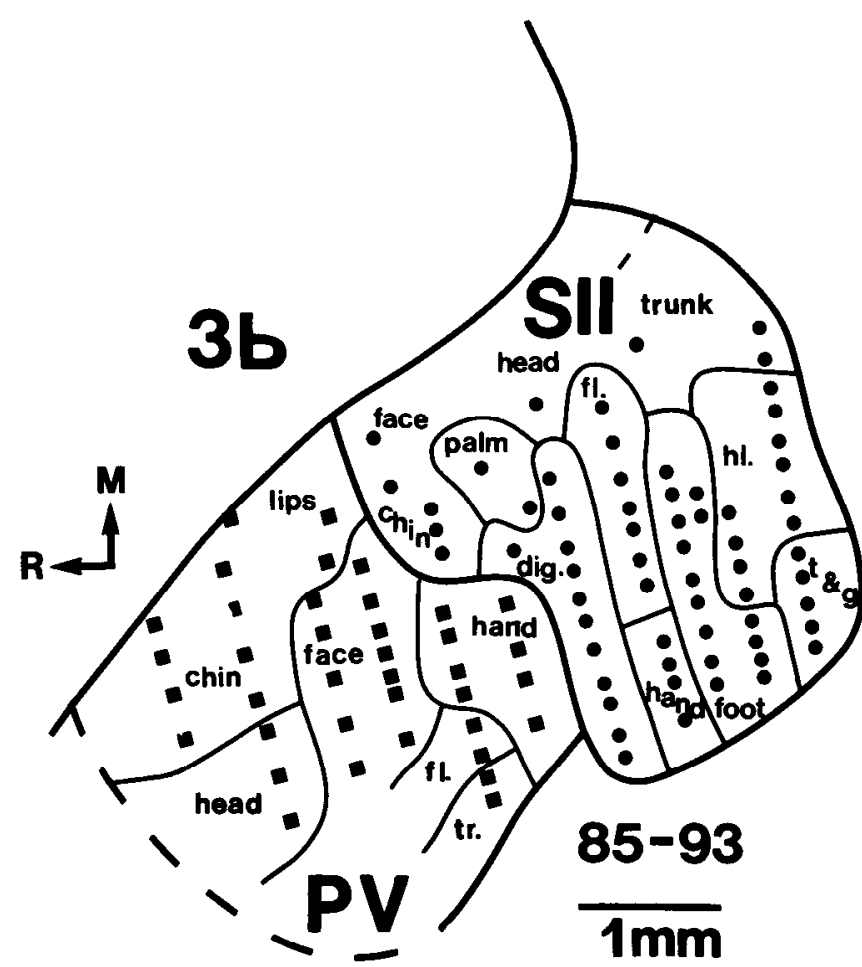

Figure 7. Somatotopic maps of S-II and PV for marmoset 85-93. $f l$, forelimb; $t r$, trunk; $h l$, hindlimb; $t$, tail. Other conventions as in previous figures.

gross somatotopic organizations within these fields that reflect those revealed by microstimulation experiments (see Gould et al., 1986). Most of the neurons labeled in the hindlimb/trunk injection in $3 \mathrm{~b}$, although located more medially, were widely scattered in M-I and SMA. This is in agreement with the proposed organization of motor cortex, where multiple representations of the same body part were found (Gould et al., 1986). Thus, a single hindlimb/trunk injection site in area $3 \mathrm{~b}$ may be projecting to several or all of the multiple representations of the hindlimb in motor cortex. Although the forclimb injection in $3 \mathrm{~b}$ did not label neurons in motor cortex, the face injection in $3 \mathrm{~b}$ did label neurons near the lateral margin of M-I, as expected.

Some of the conclusions regarding the topographic connections of area $3 b$ are supported by more limited results from a second case where injections of 2 different fluorescent tracers were closely placed in the hand region of area $3 \mathrm{~b}$ (Fig. 14B). Thus, adjoining parts of area $3 \mathrm{a}$ and area 1 contained neurons resulting from these injections in area $3 \mathrm{~b}$, and a rostral part of SMA, possibly representing the hand (see below) was labeled by one of the injections. However, labeled neurons were also present medially in areas $3 a$ and 1 , and caudally in SMA, and these neurons would be somatotopically mismatched locations.

The injection of the fluorescent dyes and, to some extent, the WGA-HRP also revealed some features of intrinsic connections in area $3 \mathrm{~b}$. Many labeled neurons were found very close to injection sites, suggesting the presence of short lateral conncctions. In addition, scattered neurons and small foci of neurons in area $3 \mathrm{~b}$ could be found labeled as far as $2 \mathrm{~mm}$ from injection sites, providing evidence for longer lateral connections (Fig. 14B).

Finally, the present cases provided some evidence about the 


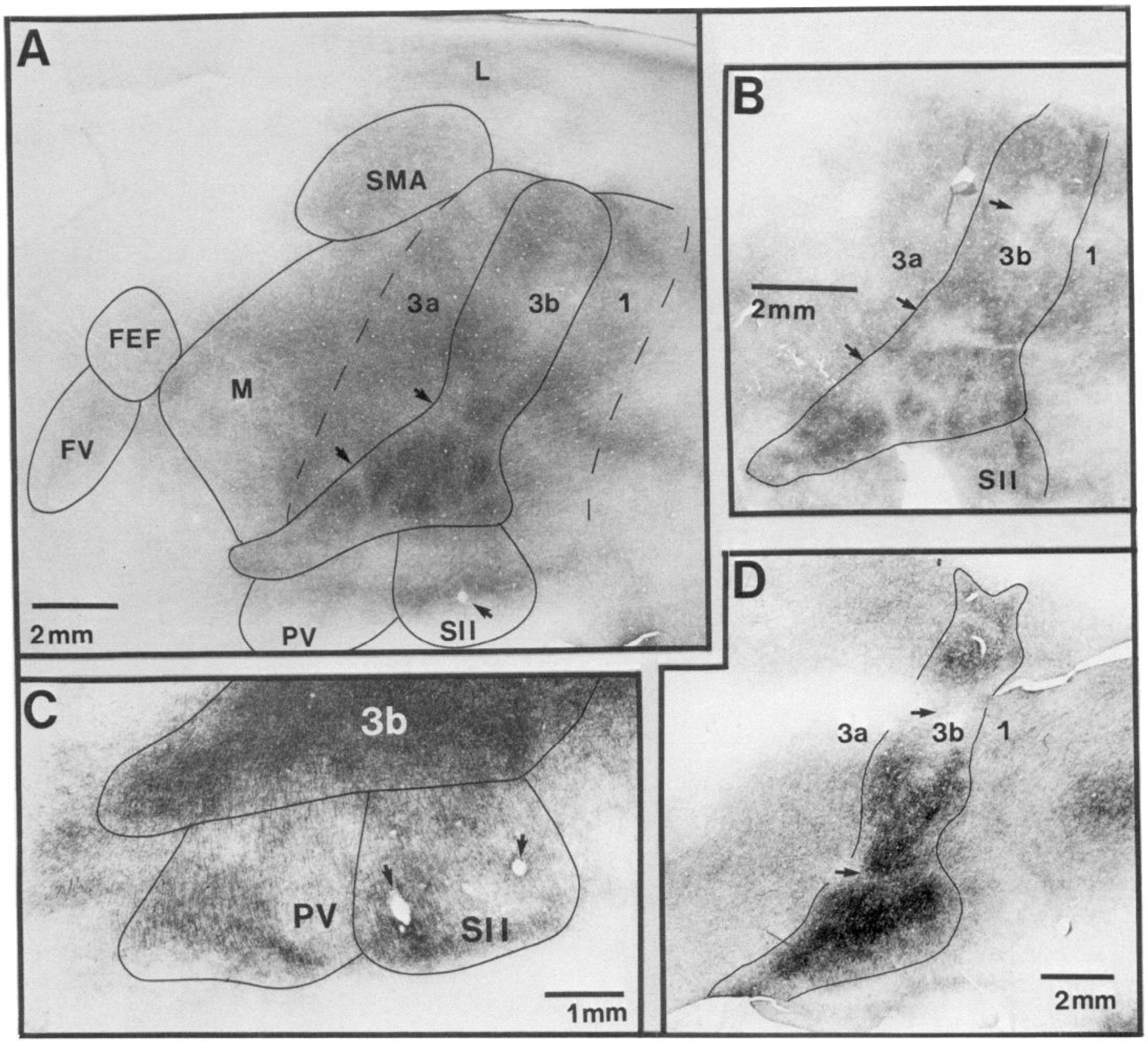

Figure 8. A, Light-field photomicrograph of cortex that has been flattened and stained for myelin. In addition to the darkly myelinated $3 \mathrm{~b}$, other cortical areas are apparent as well. Lightly myelinated areas (arrows) separate major body parts in $3 \mathrm{~b}$. The small arrow in S-II points to a micropipette puncture from an injection of WGA-HRP in S-II. $B$, Light-field photomicrograph of a myelin-stained section demonstrating the myelin-poor zones (arrows) in 3b that separate major body parts. $C$, Light-field photomicrograph of myelin-stained cortex in the lateral sulcus. The lateral portion of $3 \mathrm{~b}$ is visible here as a darkly myelinated region of cortex. S-II and PV are more moderately myelinated. Arrows in S-II point to microlesions placed at approximate physiological boundaries. $D$, Light-field photomicrograph of cortex that has been sectioned tangentially and stained for myelin. Cortical area $3 \mathrm{~b}$ stains very densely for myelin and is quite distinct from more lightly staining $3 \mathrm{a}$ rostrally and moderately staining area 1 caudally. In all figures, dotted lines mark architectonic boundaries and dashed lines indicate approximated boundaries. Conventions as in previous figures.

nature of callosal connections of area $3 \mathrm{~b}$ (Figs. 11, 12A). After strip injections in area $3 \mathrm{~b}$, transported label was found in area $3 \mathrm{~b}, \mathrm{~S}$-II, and PV of the opposite hemisphere. The callosal label in these 3 fields was very unevenly distributed. In area $3 b$, label appeared to be concentrated in regions just lateral and just medial to the representation of the glabrous hand, which had little label. The location of the transported label lateral to the hand representation coincided with a poorly myelinated zone (Fig. $8 B$ ) separating hand and face representations. The locations of some of the callosally transported label along the lateral border of area $3 \mathrm{~b}$ was not matched by any of the injection sites, and thus some callosal connections were somatotopically mismatched.

\section{The second somatosensory area, $S-I I$}

Injections of WGA-HRP in S-II of marmosets revealed interconnections with all subdivisions of anterior parietal cortex, motor and visuomotor fields of frontal cortex, $\mathrm{PV}$ and other cortex of the lateral sulcus, and medial limbic cortex. Results were obtained from 2 cases (Figs. 14B, 17), where injections 


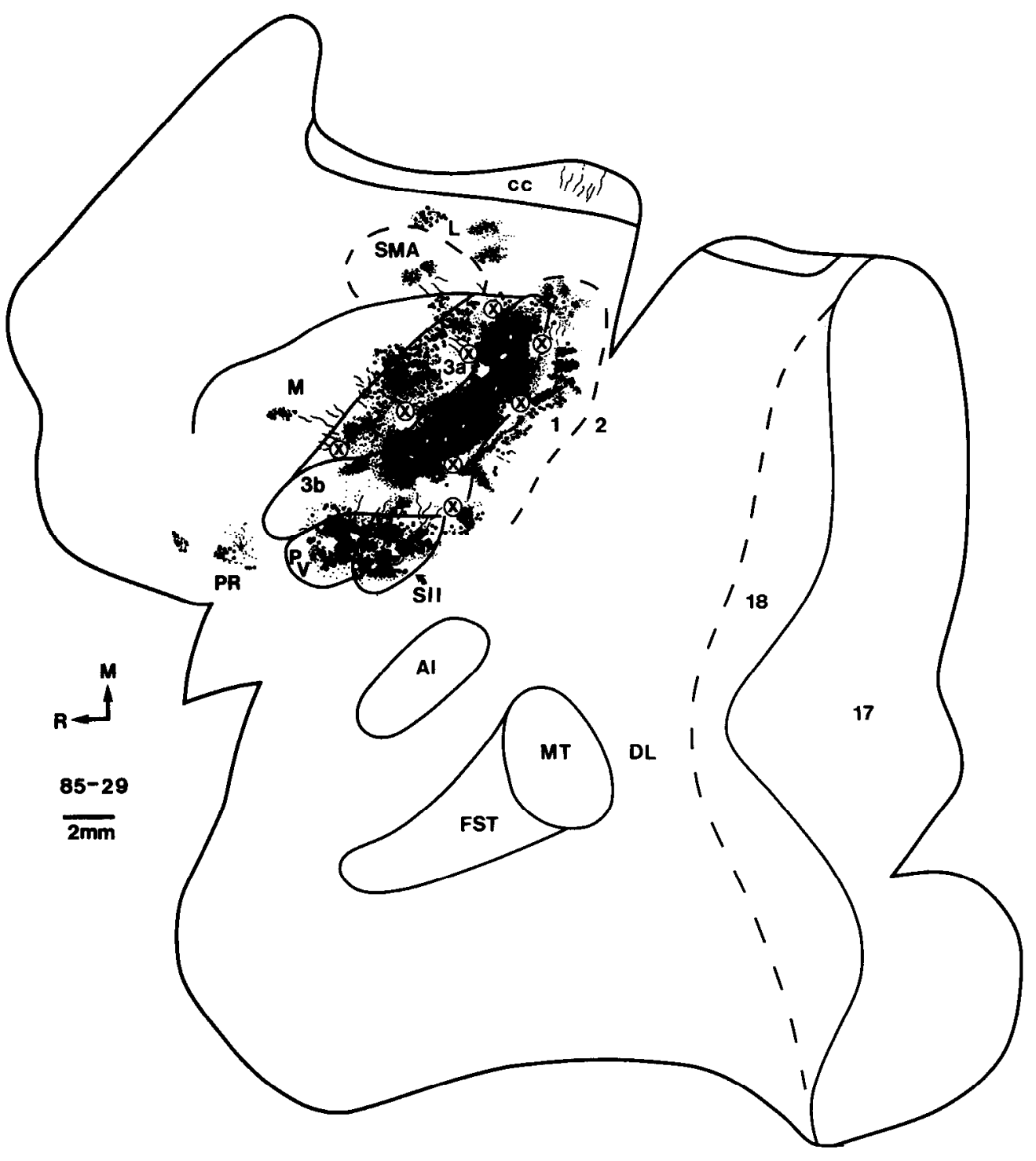

Figure 9. Distribution of label after a strip of WGA-HRP injections in the primary somatosensory area, $3 \mathrm{~b}$ in marmoset 85-29. Dense foci of anterogradely (small dots) and retrogradely (large dots) transported label are in areas 3a, 1, S-II, and PV. Sparser amounts of label are in motor cortex $(M)$, the architectonically defined supplementary motor area $(S M A)$, limbic cortex $(L)$ just medial to SMA and cortex rostral to PV. Cortex has been separated from the brain, the lateral and calcarine fissures have been opened, and cortex has been flattened in a single sheet. Circled $x$ 's mark lesions at or near physiological boundaries. Solid lines delineate architectonic boundaries, and dashed lines mark approximated boundaries. $c c$, corpus callosum; $A-I$, primary auditory field; $M T$, middle temporal visual area; $F S T$, fundal superior temporal area; 17 , primary visual area; 18 , second visual area; $R$, rostral; $M$, medial. were centered in the representation of the forelimb of S-II (Figs. $3,4)$. Both cases demonstrated prominent patches of label in area $3 b$, supporting the evidence from area $3 b$ injections that $\mathrm{S}-\mathrm{II}$ and area $3 \mathrm{~b}$ are strongly interconnected. The patches of label were within and around the midportion of area $3 \mathrm{~b}$ where the forelimb is represented, thus demonstrating somatotopic interconnections. In case 86-58 (Fig. 14B), microelectrode recordings directly demonstrated that the labeled region in area $3 \mathrm{~b}$ represents the forearm and wrist, and an injection of Fast blue in this region labeled neurons in and around the WGAHRP injection site in S-II. Other patches of label were in midportions of areas $3 \mathrm{a}$ and 1 , providing further evidence for parallel somatotopic representations in these fields. The region of label in area 1 extends into adjoining parts of area 2, suggesting that area 2 also contains a parallel somatotopic representation.

Some label caudal to S-II in both cases may have been in lateral extensions of areas 1 and 2. If so, this label would appear to be out of somatotopic register since this portion of areas 1 and 2 would be expected to represent the face. Possibly the label was a consequence of some involvement of the face representation in the injection, but this contention is not supported by any substantial labeling of the face region of area 3b. Another possibility is that the label is in another field lateral to areas 1 and 2 .

In both cases, foci of label in PV were located in more "lateral" (deeper) levels in PV. Other foci of label just rostral to PV could be part of motor cortex or part of another field in rostroventral parietal cortex, PR, which also has inputs from area $3 \mathrm{~b}$. Interconnections were demonstrated with both M-I and SMA. Label in M-I was in several scattered foci just rostral to the label in area $3 \mathrm{a}$ in case 86-58 (Fig. 14B) and more lateral in case 86-55 (Figs. 17, 18). Other foci in both cases were located more laterally, with the most lateral label possibly outside of M-I and PR. Label was largely restricted to the middle to rostral portion of SMA, a location that is compatible with the somatotopic matching of connections between forelimb representations. Surprisingly, the S-II injections also resulted in label in the frontal eye field (FEF) and the frontal visual area (FV) fields that appear to be directly involved in producing eye movements (Kaas and Krubitzer, 1988).

Like $3 b, S-I I$ also had interconnections with limbic cortex medial to SMA. Other label was lightly scattered in cortex be- 
Figure 10. Dark-field photomicrograph of cortex that has been sectioned parallel to the cortical surface and reacted for HRP in marmoset 85-29. The long dark strip surrounded by the white haze in area $3 \mathrm{~b}$ is the core of the injection site and the surrounding local uptake zone. Patches of anterograde label are in areas $3 \mathrm{a}$ and 1 immediately rostral and caudal to $3 \mathrm{~b}$. Lateral to $3 \mathrm{~b}, \mathrm{~S}-\mathrm{II}$ and PV also contain dense patches of label. Solid white lines mark architectonic boundaries. Small arrows point to electrolytic lesions placed at physiological boundaries. Other conventions as in previous figures.

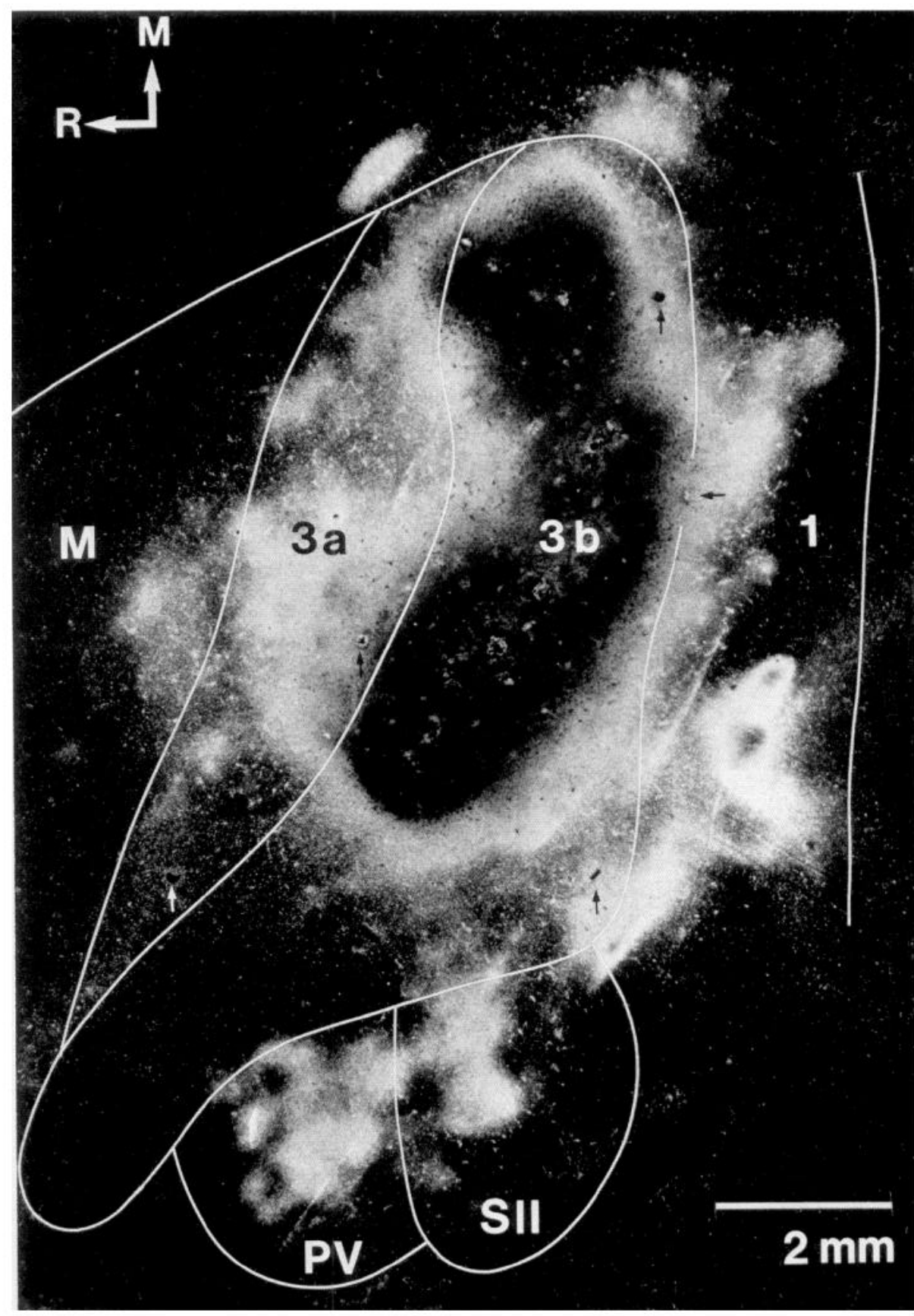

tween primary auditory cortex and the middle temporal visual area (MT), but this label was in somewhat different locations in the 2 cases.

Both cases with S-II injections revealed callosal connections with S-II and PV of the other hemisphere (Figs. 12B, 16, 19). The label was patchy and scattered over much of S-II and PV. Additional foci of callosally transported label were found in areas 1, 3b, and M-I of case 86-55 (Fig. 19). The label in these fields was in locations where the face and hand representations might join and where less dense myelination occurs in area $3 \mathrm{~b}$. The injection sites in S-II involved cortex representing the forelimb. Parts of the forearm representation have been found between the hand and face in area $3 \mathrm{~b}$ of some primate species and other mammals (see Kaas, 1983), but not in marmosets (Carlson et al., 1986). Other label was at the lateral margin of M-I, possibly in PR.

\section{Laminar distribution of connections of $S-I$ and $S-I I$}

The location of cell bodies and axon terminals in separate lamina of different cortical fields after injections in 3b and S-II was approximated from relative depths from the surface in the tangentially sectioned tissue. Other observations of laminar patterns of connections were obtained from one case with an S-II injection that was sectioned coronally. Strip injections in S-I resulted in dense patches of label in cortical areas S-II and PV. In these regions, superficial and deeper layers contained the labeled cell bodies, while middle sections, including layer IV contained anterogradely labeled terminations. Thus, $3 \mathrm{~b}$ has 


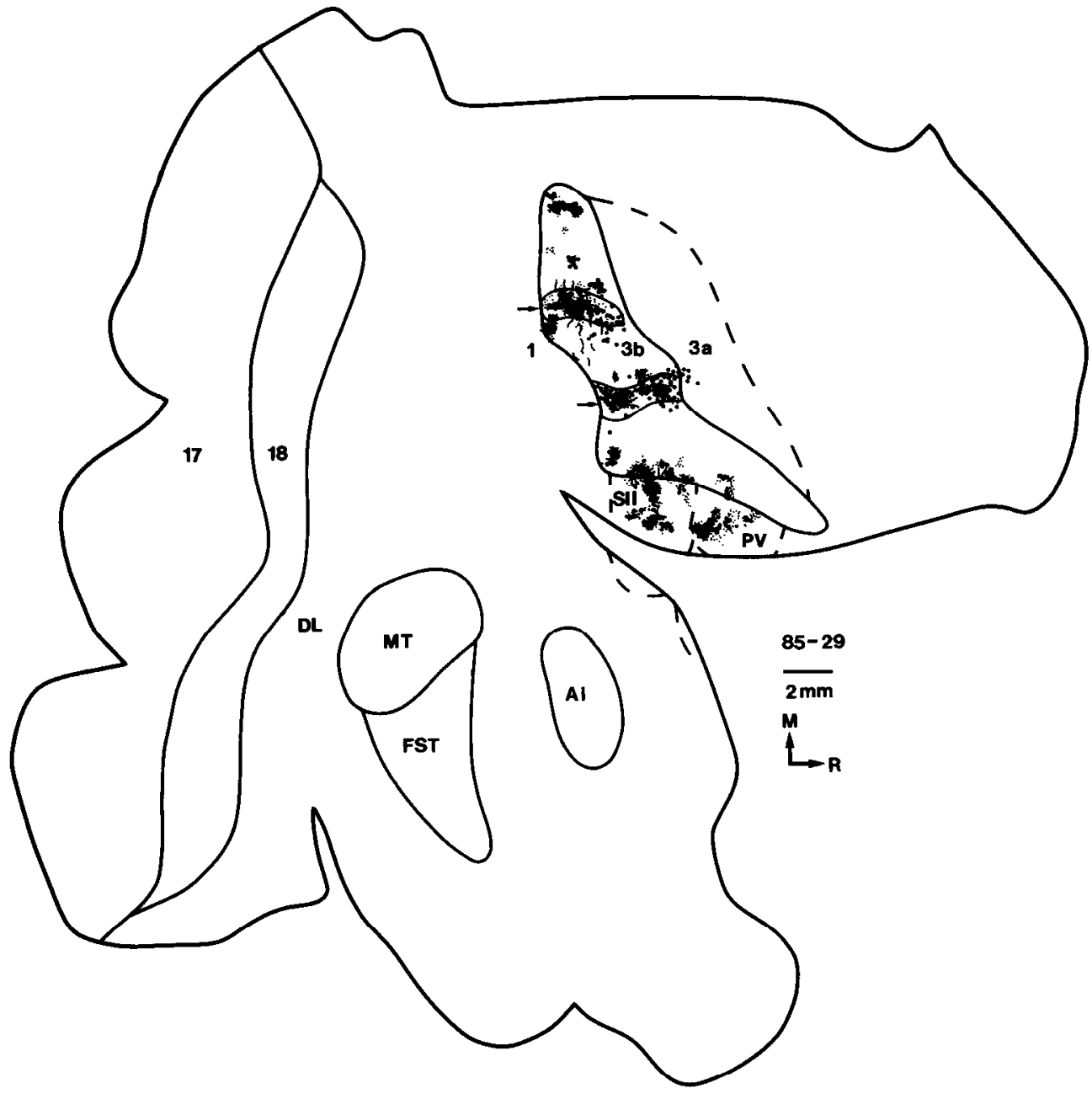

Figure 11. Distribution of transported label in the hemisphere contralateral to area $3 \mathrm{~b}$ injections (see Fig. 9) in marmoset 85-29. Although an entire mediolateral strip of $3 b$ in the opposite hemisphere was injected, label in this hemispherc is only in limited portions of $3 \mathrm{~b}$. Patchy label is also observed in $\mathrm{S}-\mathrm{II}$ and PV. Conventions as in previous figures. "feedforward" (Van Essen and Maunsell, 1983) connections with S-II and PV. The hierarchical relationship between $3 \mathrm{~b}$ and S-II determined in tangentially sectioned cortex was verified in one casc where S-II was injected and cortex was sectioned coronally. Labeled cell bodies and axon terminals in $3 \mathrm{~b}$ were confined to layers II, III, V, and VI and absent from layer IV.

The relationship between S-II and PV was not obvious in the case sectioned coronally because this case, with a relatively short survival time (12 hr), produced little label in PV. However, in cases with injections in S-II in tangentially sectioned cortex, labeled cell bodies in PV were in both deep and superficial layers, and axon terminals were distributed across cortical layers including layer IV. This suggests that $\mathrm{PV}$ is at a similar or higher hierarchical level as S-II.

\section{Discussion}

The present study presents microelectrode mapping data on the somatotopic organization of 3 areas of somatosensory cortex in marmosets: area $3 \mathrm{~b}$, or S-I proper, S-II, and a newly described field in primates, the parietal ventral area, PV. Injections of anatomical tracers into electrophysiologically identified locations in area $3 \mathrm{~b}$ and S-II demonstrated patterns of somatotopic organization in other fields, including areas $3 \mathrm{a}$ and 1 , and im- plicate a number of other regions of cortex in the processing of somatosensory information (Fig. 20).

The responsiveness and somatotopic organization of somatosensory and motor cortex

Area $3 b$

Our microelectrode recordings from area $3 \mathrm{~b}$ of marmosets produced results that are highly consistent with findings previously reported for marmosets and the closely related tamarins (Carlson et al., 1986; Huerta et al., 1986; Krubitzer and Kaas, 1986, 1988; Wall et al., 1986; Garraghty et al., 1989). Most or all recording sites in area $3 \mathrm{~b}$ could be activated by lightly touching the skin or brushing the hairs of the body surface, and inputs from different body regions activated specific locations in the field to form a highly ordered representation of the body surface. The representation is organized so that the hindlimb, trunk, forelimb, and face form a mediolateral sequence in cortex, and the glabrous digits are rostral to the palm. This basic pattern of representation corresponds to that found in area $3 b$ of other species of monkeys, and in S-I of prosimian primates and a wide range of other mammals (see Kaas, 1983, for review). The similarity in the organization of S-I as described in nonprimates and prosimians and area $3 \mathrm{~b}$ of monkeys is part of the evidence 

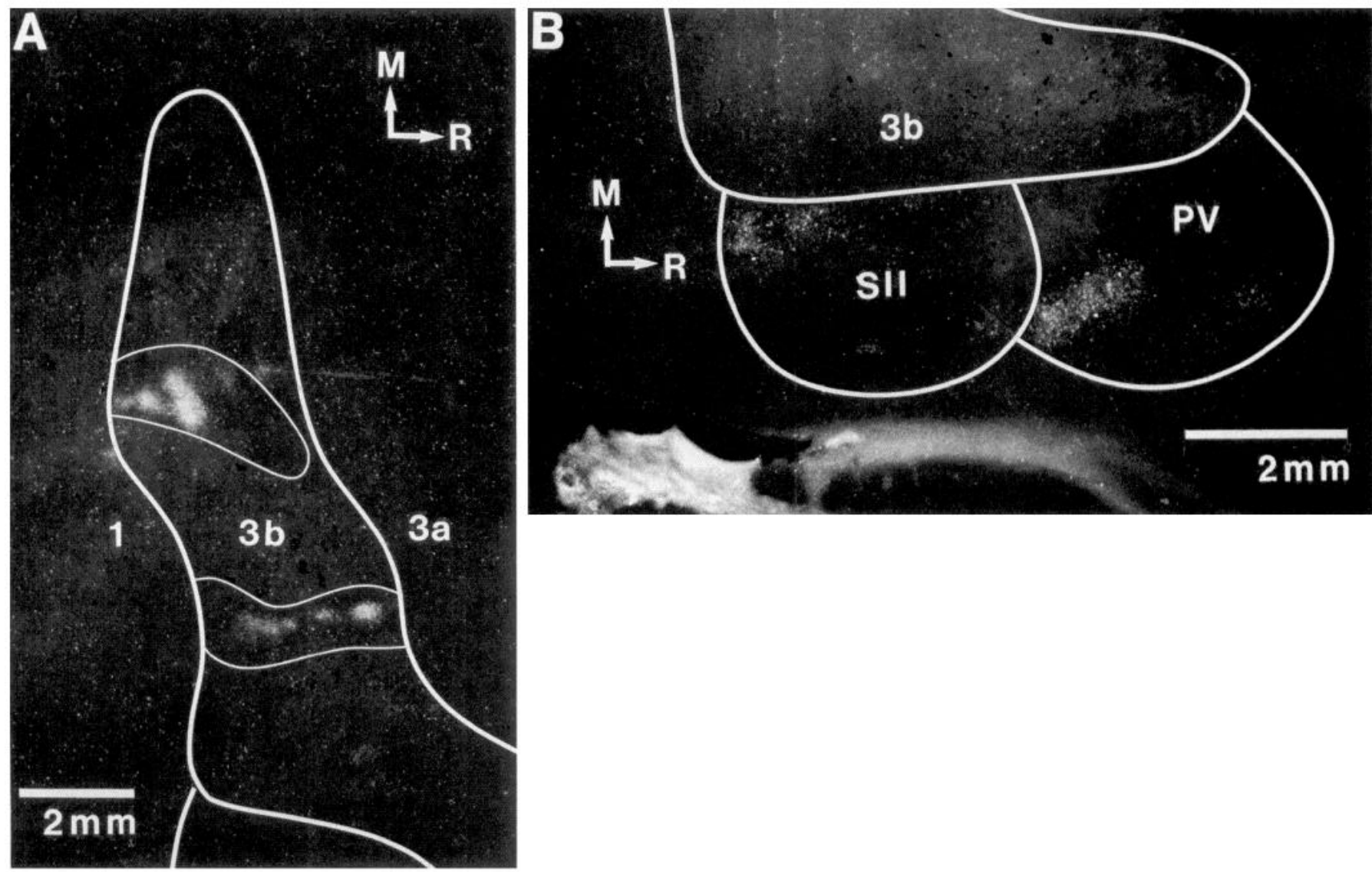

Figure 12. A, Dark-field photomicrograph of labeled cell bodies and axon terminals in 3b after strip injections of WGA-HRP in $3 \mathrm{~b}$ in the opposite hemisphere in marmoset 85-29. Note that only the portions of $3 \mathrm{~b}$ that correspond to the myelin-poor zones contain label. Thick white lines mark architectonic boundaries and thin white lines in 3b mark myelin-poor zones. B, Dark-field photomicrograph of label in areas S-II and PV after injection in S-II in the opposite hemisphere in marmoset 86-55. Solid white lines mark architectonic boundaries. Other conventions as in previous figures.

that the fields are homologous, and thus we use the term S-I or S-I proper for area $3 \mathrm{~b}$ of monkeys.

Areas $3 a, 1$, and 2

As previously reported (Carlson et al., 1986), areas 3a and 1 responded poorly to cutaneous stimuli. Neurons in area $3 \mathrm{a}$ responded to taps and body movements, and usually light touch and the movement of hairs were not effective stimuli. These responses are consistent with evidence that area $3 \mathrm{a}$ in other primates is activated by thalamic inputs related to muscle spindle receptors (Phillips et al., 1971; Lucier et al., 1975; Merzenich et al., 1978; Nelson et al., 1980; Maendly et al., 1981; see Wiesendanger and Miles, 1982, and Kaas and Pons, 1988, for review), although some cutaneous driving occurs, possibly from cortical inputs from area 3b (Jones and Powell, 1969a; Jones and Wise, 1977; Vogt and Pandya, 1978; see Kaas and Pons, 1988 , for review). Since area 3 a does not receive cutaneous input from the ventral posterior nucleus of the thalamus (see Kaas and Pons, 1988), cutaneous activation is likely to be either directly or indirectly from area $3 \mathrm{~b}$. In addition, both the activation patterns of $3 \mathrm{a}$ in tamarins (Carlson et al., 1986) and other primates (Merzenich et al., 1978; Nelson et al., 1980) and the pattern of the topographic connections with area $3 \mathrm{~b}$ in the present investigation indicate that area $3 \mathrm{a}$ contains a representation of the body parallel to that found in area $3 \mathrm{~b}$ (Fig. 15).
Since area 1 was relatively unresponsive to light touch or even more intense cutaneous stimuli in marmosets, while area 1 is highly responsive to cutaneous stimulation in other monkeys (Merzenich et al., 1978; Nelson et al., 1980; Sur et al., 1982; Felleman et al., 1983; Cusick et al., 1985), there is some uncertainty if the field caudal to area $3 \mathrm{~b}$ in marmosets is area 1 of other monkeys (see Carlson et al., 1986). In the present investigation, the connection patterns with areas $3 \mathrm{~b}$ and S-II demonstrate a somatotopic organization within "area 1" of marmosets that is in parallel with the area $3 \mathrm{~b}$ representation, as is found by activation patterns in other monkeys. Another possibility is that the region caudal to $3 \mathrm{~b}$ is area 2 . However, unlike area 1 , area 2 does not have dense topographic connections with area $3 \mathrm{~b}$ and S-II in other monkeys (Jones and Powell, 1969a; Jones and Wise, 1977; Jones et al., 1978; Vogt and Pandya, 1978; Friedman et al., 1986; Pons and Kaas, 1986) and the caudally adjacent field to $3 \mathrm{~b}$ in marmosets does have very dense connections with both $3 \mathrm{~b}$ and S-II. Thus, our results are consistent with the previous contention (Carlson et al., 1986) that the strip of cortex caudal to area $3 \mathrm{~b}$ in marmosets is area 1 of other primates, but this area 1 has less secure driving by cutaneous stimuli.

There is no compelling evidence that marmosets have an area 2. Cortex caudal to area 1 in other monkeys, generally described as area 2, contains a representation of the body that is also 


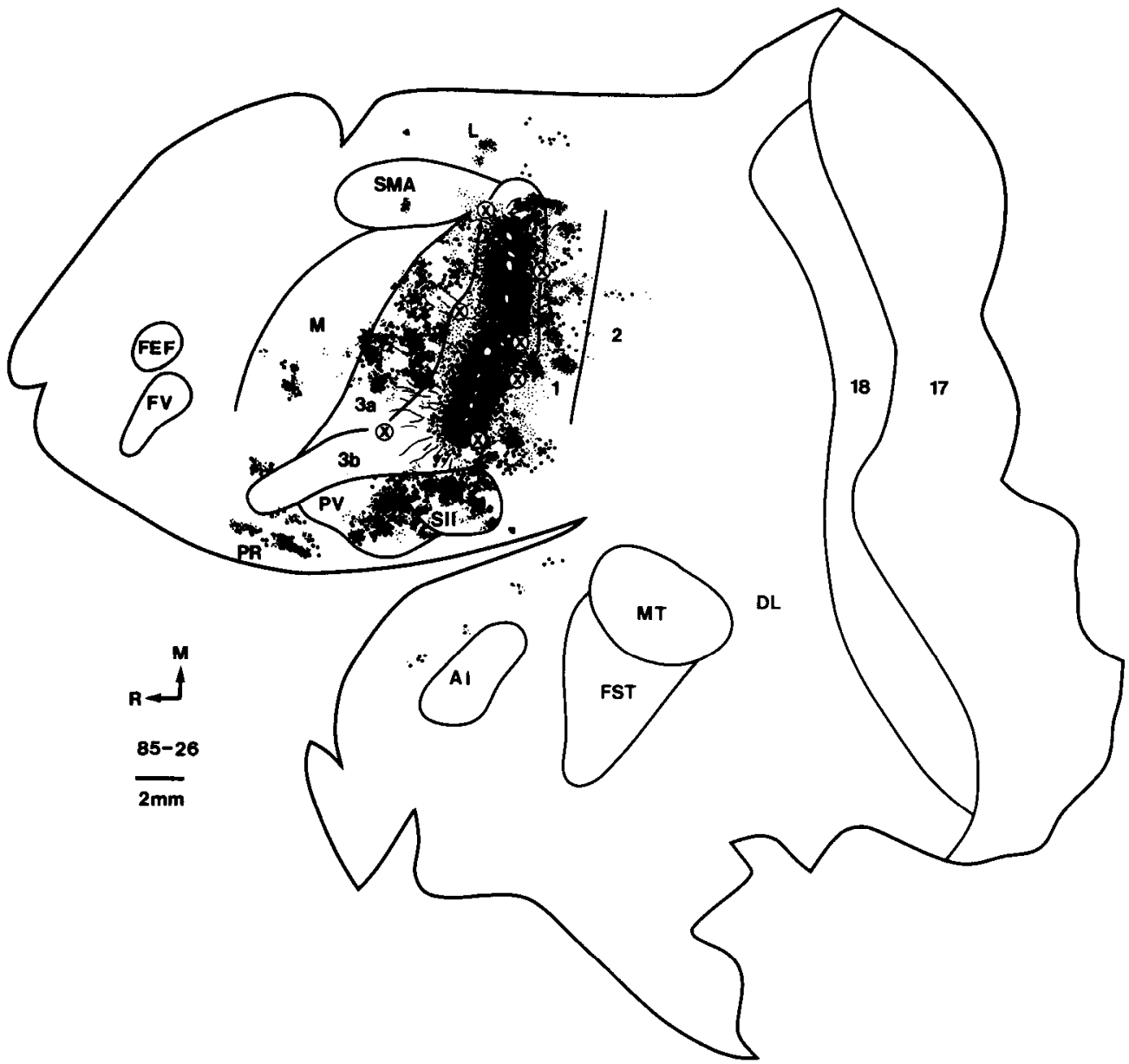

Figure 13. Areal distribution of label after a strip of WGA-HRP injections in the primary somatosensory area, $3 \mathrm{~b}$ in marmoset 85-26. As in case 85-29, transported label is predominantly in areas 3a, 1, S-II, and PV. Sparser amounts of label are in SMA, limbic cortex, M, cortex rostral to PV, area 2, and cortex lateral to S-II. Conventions as in previous figures. parallel to that in area $3 \mathrm{~b}$ (e.g., Merzenich et al., 1978), but the organization of the representation is not known in detail. Parts of area 2 are highly responsive to cutaneous stimuli in macaque monkeys, and microelectrode maps in these areas reveal repetitions in the representation and other complexities that are not found in areas $3 \mathrm{~b}$ and 1 (Pons et al., 1985a, b). Other parts of area 2 in macaque monkeys and area 2 in owl monkeys (Merzenich et al., 1978) and squirrel monkeys (Sur et al., 1982) are largely activated by deep receptors, perhaps muscle spindle receptors via thalamocortical inputs from the ventroposterior superior nucleus (see Cusick et al., 1985). The partial activation of area 2 by cutaneous receptors probably depends on connections from areas $3 \mathrm{~b}$ and 1 (Jones and Powell, 1969a; Jones and Wise, 1977; Jones et al., 1978; Vogt and Pandya, 1978; Pons and Kaas, 1986), although sparse inputs from the ventroposterior nucleus of the thalamus (Pons and Kaas, 1985) could be effective in activating area 2.

In marmosets, cortex in the location of area 2 differs from area 2 of other monkeys in 2 major ways. First, there was no obvious driving by inputs from either cutaneous or deep peripheral receptors. Second, there appears to be almost no connections with area $3 \mathrm{~b}$. However, present results indicate that the area 2 region does have at least sparse connections with S-II, and portions of area 2 have connections with S-II in macaque monkeys (Vogt and Pandya, 1978; Friedman, 1983; Friedman et al., 1986; Pons and Kaas, 1986). Furthermore, injections in the part of S-II representing the forelimb labeled a midportion of the "area 2" region approximately where the forelimb is represented in area 2 of other monkeys. Thus, the "area 2" region in marmosets may have a somatotopic organization similar to that of area 2 of other monkeys. Overall, we favor the possibility that the cortex we have termed area 2 in marmosets is homologous to area 2 described in other monkeys because of its relative position, topography, and interconnections with S-II. However, unlike area 2 of other monkeys, this area in marmosets lacks activating inputs from area $3 \mathrm{~b}$. If area 2 exists in marmosets, it should have other characteristics of area 2 in other monkeys such as cortical inputs from area 1 and thalamic inputs from the ventroposterior superior nucleus, but such information is presently lacking.

\section{$S-I I$}

Our microelectrode recordings revealed the somatotopic organization of S-II in detail. The border region of area $3 b$ with S-II was congruent so that the representation of the midline of the upper face joined the 2 fields. The fore- and hindlimbs were displaced from the $3 \mathrm{~b}$ border and the trunk was caudal in S-II. Overall, the representation is of the type termed "erect," since the head adjoins S-I and the feet point away from S-I, rather than "inverted," with the feet adjoining S-I and the head displaced away from the border (see Nelson et al., 1979, for review). Basically similar organizations have been reported for other monkeys (owl monkeys: Cusick et al., 1989; squirrel monkeys: Cusick and Manning, 1988; macaque monkeys: Whitsell et al., 
Figure 14. A, Patterns of label resulting from 3 different tracers injected into separate physiologically defined body part representations in area $3 \mathrm{~b}$ in marmoset 85-51. Fast blue was injected in the hindlimb representation of area $3 \mathrm{~b}$; WGA-HRP was injected in the forelimb representation; and fluoro-gold was injected in the face representation. $B$ Cortical reconstruction of a WGA-HRP injection in the hand representation of $\mathrm{S}-\mathrm{II}$ and injections of Fast blue and diamadino yellow in the hand and wrist region of area $3 \mathrm{~b}$ in marmoset 86-58. Label in 3b from the S-II injection is predominantly in the region of the hand representation. The injection in S-II also labeled areas $3 \mathrm{a}$ and 1 at a similar mediolateral level as the label in $3 \mathrm{~b}$. Motor cortex, SMA, area 2, limbic cortex, and cortex lateral to SII also contain label from the S-II injection. Finally, the frontal eye field $(F E F)$ and the frontal visual area $(F V)$ contain labeled cell bodies and axon terminals as well. Connections from the $3 \mathrm{~b}$ injections are similar to those described for other cases (Figs. 9 and 13). Conventions as in previous figures.
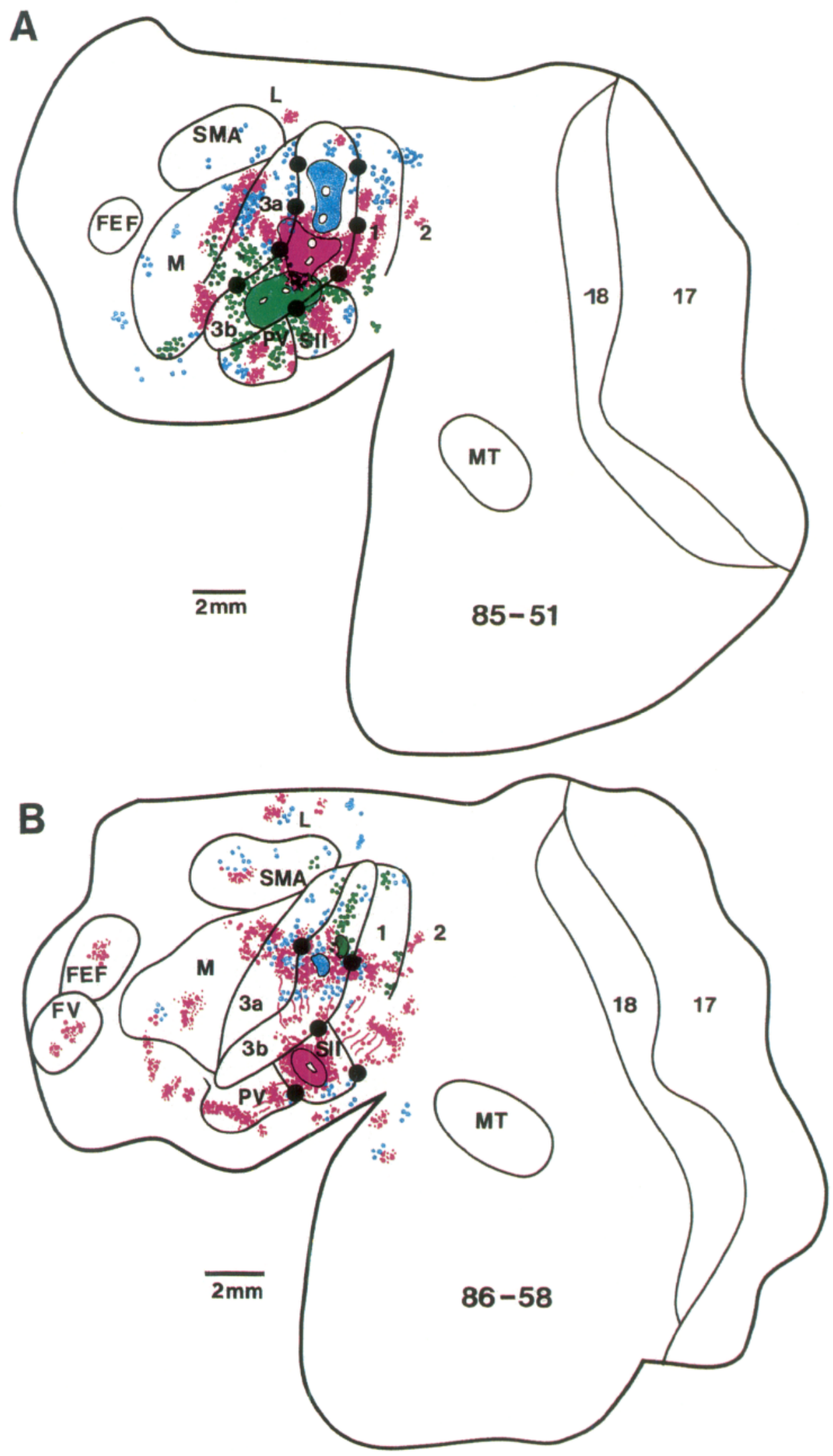


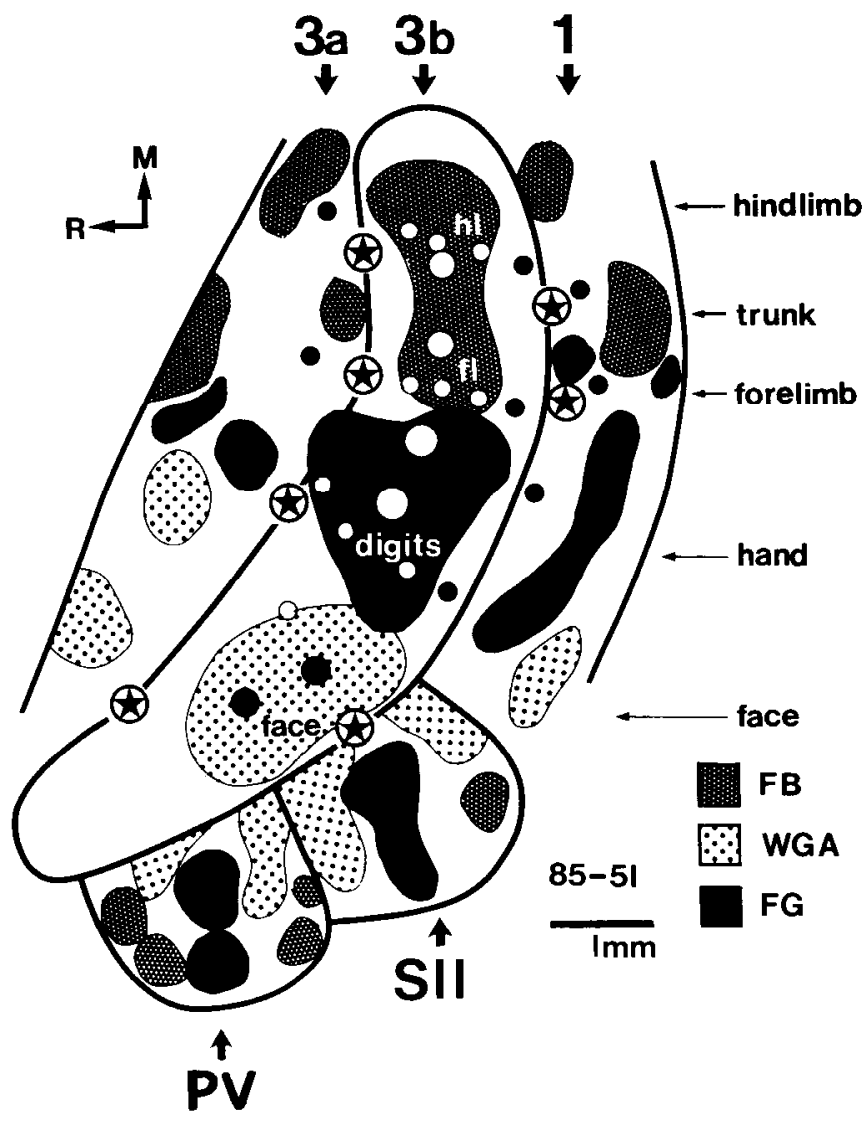

1969; Robinson and Burton, 1980; Pons et al., 1987, 1988), prosimian galagos (Burton and Carlson, 1986), tree shrews (Sur et al., 1981), and a wide range of nonprimate species (see Nelson et al., 1979, for review). Early suggestions that S-II is "inverted" seem to be in error.

The representation in S-II was somatotopically simple, as reported in most mammals. We did not observe an embedded zone of neurons within the field such as the "rostral complex zone," as reported by Robinson and Burton (1980) in macaque monkeys, or an embedded zone of neurons with higher thresholds of activation by deep receptors within S-II, as reported in owl monkeys (Cusick et al., 1989). In recent studies of S-II in macaque monkeys (Pons et al., 1987, 1988) such complex zones were not noted.

The results indicate that in marmosets S-II immediately adjoins area $3 \mathrm{~b}$. In other mammals such as mice (Carvell and Simons, 1986), rats (Welker and Sinha, 1972), squirrels (Nelson

Figure 15. Locations of injections of 3 separate tracers relative to a map of $3 \mathrm{~b}$ in marmoset $85-51$. Fast blue $(F B)$ was injected into the hindlimb and trunk representations and spread slightly into the forelimb representation; WGA-HRP (WGA) was injected in the representation of the digits; and fluoro-gold $(F G)$ was injected in the face representation. Previous investigations in tamarins (Carlson et al., 1986) demonstrate the sequence of representation in $3 \mathrm{~b}$ from hindlimb laterally to face medially (right portion of figure) and limited microelectrode mapping in this case is in good agreement with previous studies. Connection patterns from $3 \mathrm{~b}$ demonstrate topographic organization of adjacent fields including areas $3 \mathrm{a}, 1, \mathrm{~S}-\mathrm{II}$, and PV. $f l$, forelimb; $h l$, hindlimb. Other conventions as in previous figures.

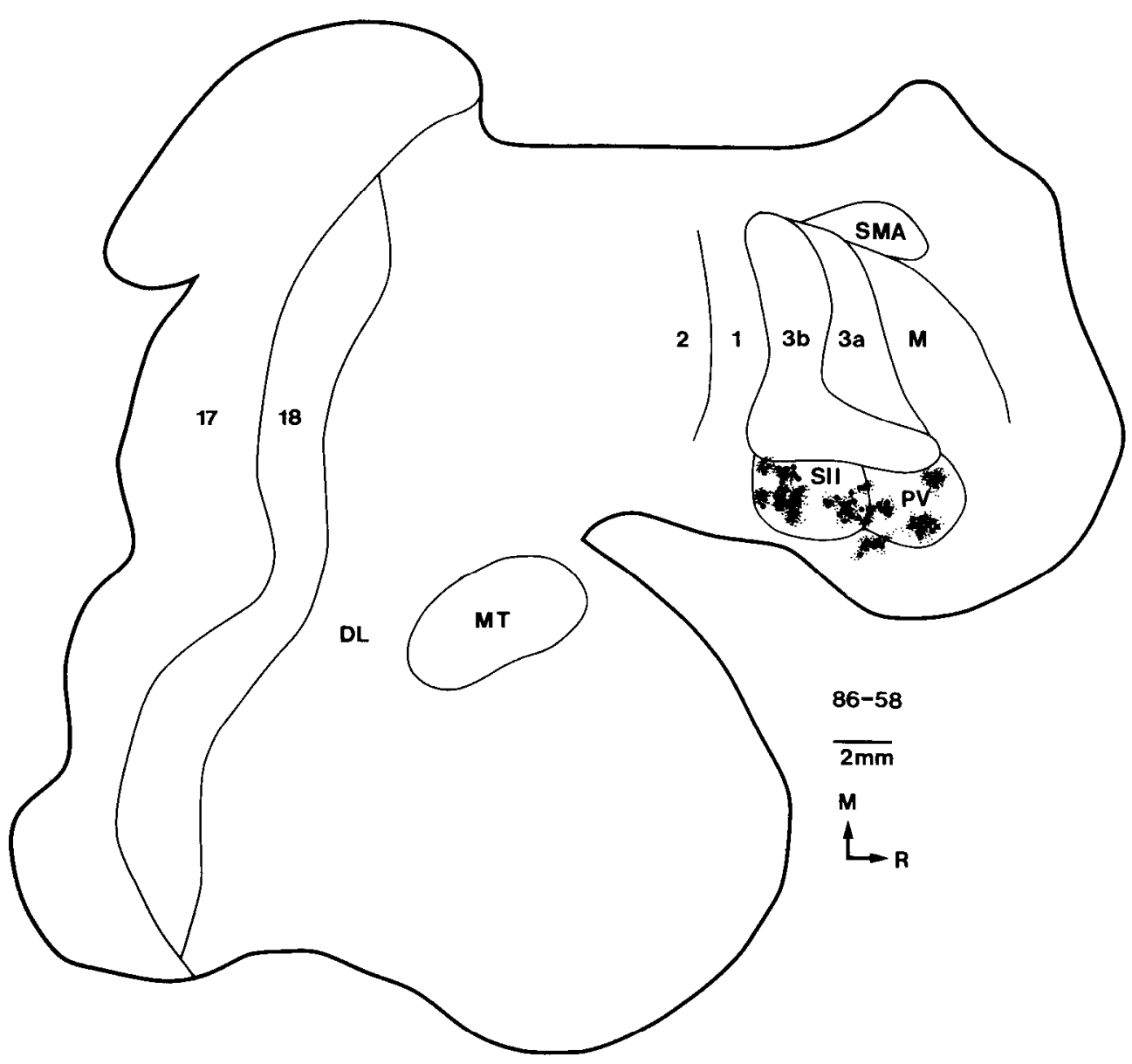

Figure 16. Distribution of callosally transported label after injections of WGA-HRP in S-II of the opposite hemisphere (Fig. 17B) in marmoset 8658. Callosal label resulting from a contralateral S-II injection is found in dense patches in S-II and PV, and in a sparse patch in cortex just lateral to PV. Conventions as in previous figures. 


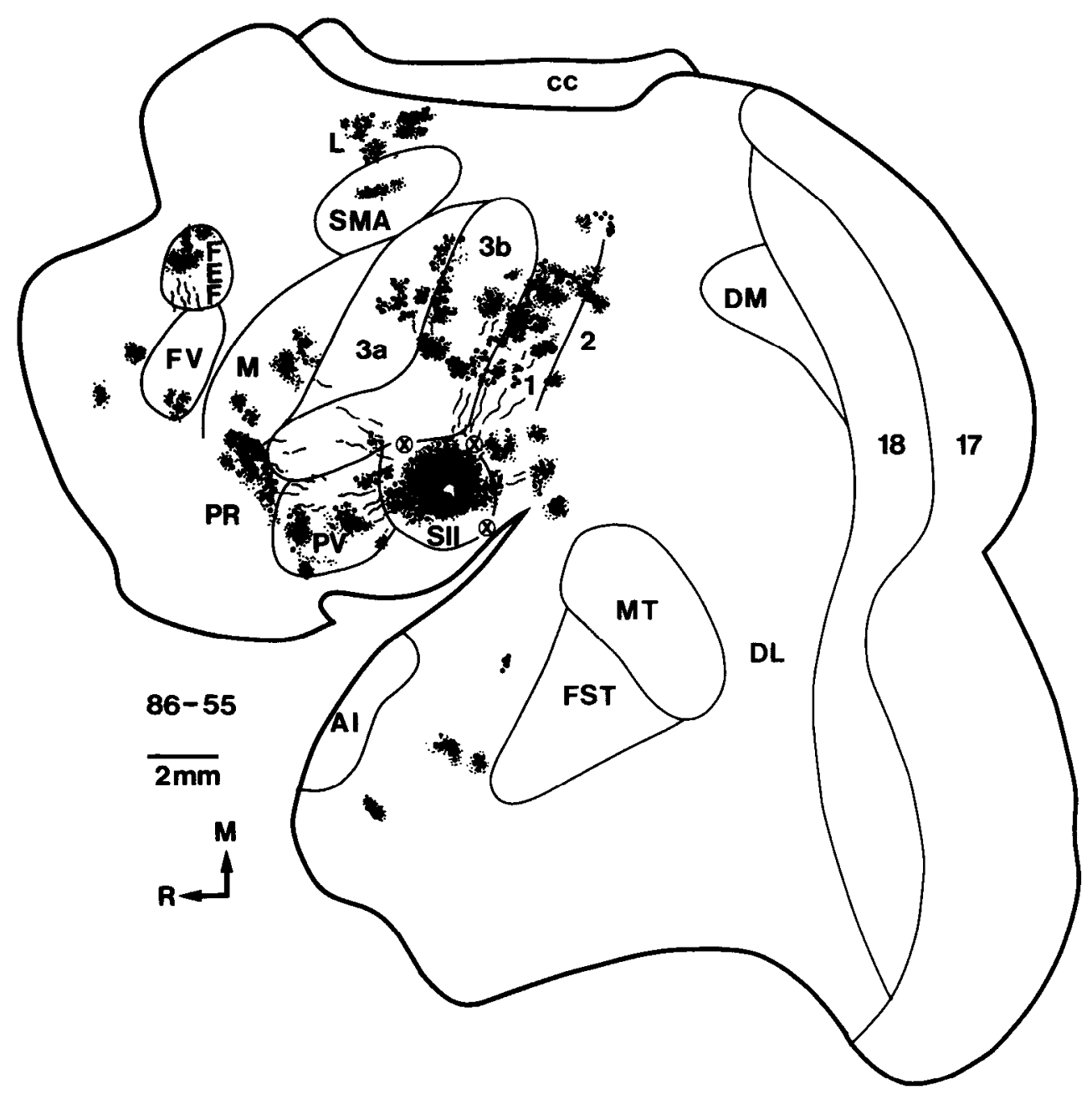

Figure 17. Areal distribution of label after an injection of WGA-HRP in S-II in marmoset $86-55$. The injection is centered in the representation of the forelimb and is completely within S-II. Dense patches of labeled cell bodies and axon terminals are in $3 \mathrm{~b}, 3 \mathrm{a}$, area 1 , PV, cortex just caudal to S-II and motor cortex. I abel is also in SMA, limbic cortex $(L)$, just rostral to FST, in FEF, $\mathrm{FV}$, and cortex just rostral to FV. Conventions as in previous figures.

et al., 1979; Krubitzer et al., 1986), agutis (Pimentel-Souza et al., 1980), porcupines (Lende and Woolsey, 1956), opossums (Pubols, 1977), cats (Haight, 1972; Burton et al., 1982; Clemo and Stcin, 1983), racoons (Herron, 1978), tree shrews (Sur et al., 1981), and even prosimian galagos (Burton and Carlson, 1986), S-II adjoins the representation of the upper face of S-I, and $S$-I of these mammals is a single representation of cutaneous receptors, the apparent homolog of the single representation of cutaneous receptors in area $3 \mathrm{~b}$ of monkeys (Kaas, 1983). The similar relation of S-II to S-I in nonprimates and galagos and area $\mathbf{3 b}$ and $\mathrm{S}-\mathrm{II}$ in marmosets supports the contention that area $\mathbf{3 b}$ is S-I. However, S-II is commonly portrayed as adjoining area 2 (Fig. 20), area $1+2$, or area 1 in both Old World and New World monkeys (e.g., Robinson and Burton, 1980; Friedman et al., 1986; Cusick et al., 1989). In owl monkeys, where there was some uncertainty in experimental results, S-II either directly borders area $3 \mathrm{~b}$ or at most, a narrow $1-\mathrm{mm}$-wide strip of area 1 separates the 2 fields (Cusick et al., 1989). These different interpretations of the relation of $3 b$ to $S$-II raise the possibility that S-II had become displaced in cvolution from the $3 \mathrm{~b}$ border in some, but not other, primate lines. However, because the relation of area $3 b$ to $S-I I$ in monkeys has not been a carefully examined issue, we favor the more conservative viewpoint that $S$-II directly borders $S$-I or area 3 b in all mammals and that no change in relative position has taken place.
The present results demonstrate for the first time in a primate that interconnections of S-I (3b) with S-II are at least roughly homotopic (Fig. 15). Previously, Friedman et al. (1980) described a topographic pattern of projections to the S-II region from sites across different mediolateral levels of area $3 \mathrm{~b}, 1$, and 2 , the traditional "S-I" of macaque monkeys. Since these fields have parallel representations that all project to $\mathrm{S}-\mathrm{II}$, the results provided a projection map that is in good somatotopic register with microelectrode maps (Robinson and Burton, 1980; Pons et al., 1987, 1988). Evidence for homotypical connections between S-I and S-II has been previously presented for cats (Jones and Powell, 1968; Manzoni et al., 1979; Burton and Kopf, 1984; Alloway and Burton, 1985), tree shrews (Weller et al., 1987), and squirrels (Krubitzer et al., 1986). Such matching of connections would be expected, of course, especially in monkeys where activation of S-II, and hence the somatotopic organization, depends on inputs from anterior parietal cortex (Pons et al., 1987; Garraghty et al., 1988, 1989).

\section{PV area}

Our microelectrode recordings revealed a systematic representation of the body surface in cortex adjoining area $3 \mathrm{~b}$ immediately rostral to S-II. We call this area the parietal ventral area (PV) because of the ventral location in the parietal lobe and because it is a probable homolog of the field in the same position 


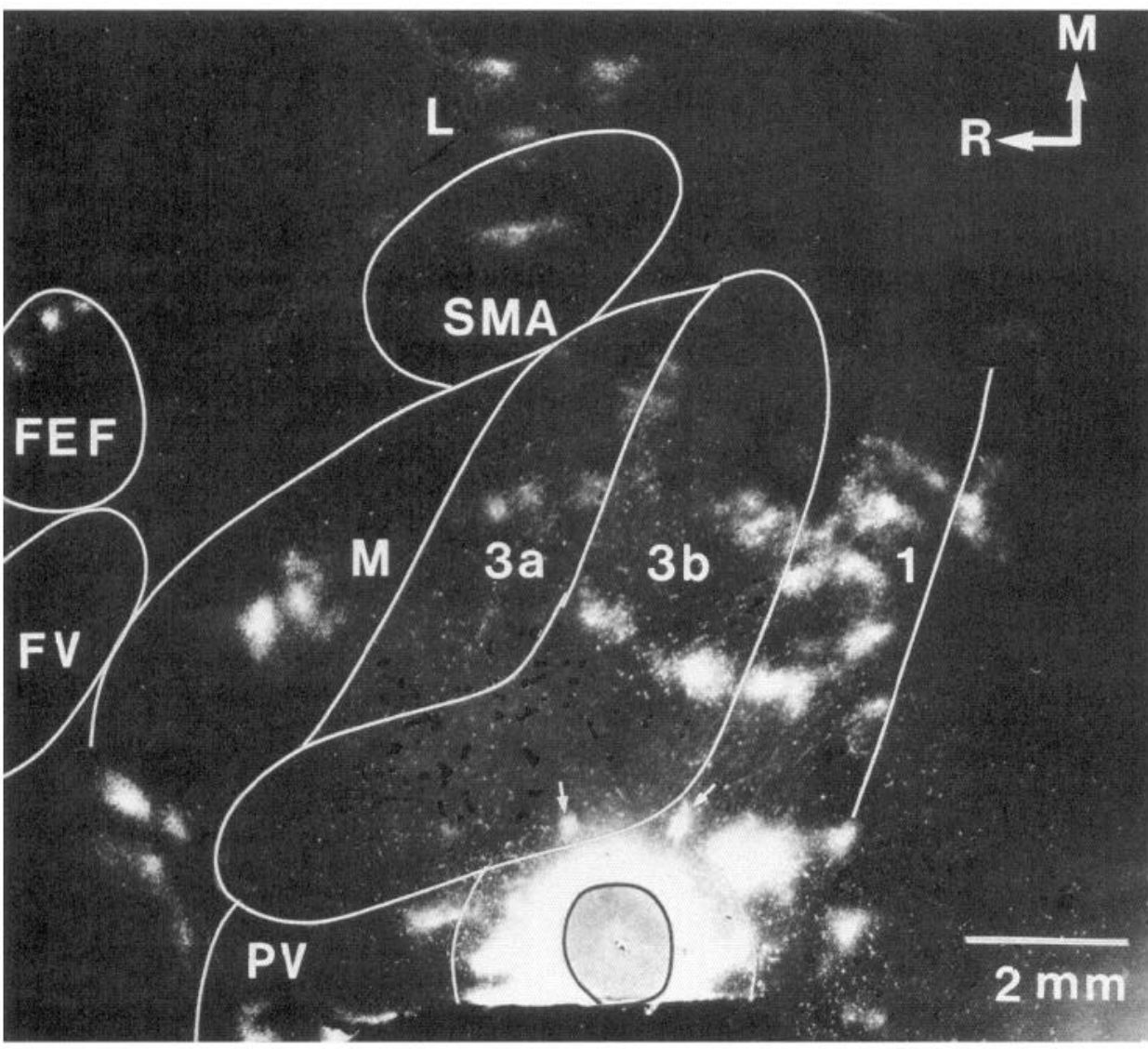

Figure 18. Dark-field photomicrograph of an injection in S-II and transported label in marmoset 86-55. The dark circle and white halo in S-II is the injection core and local uptake zone of the injection. Transported tracer (white patches) is found in $\mathrm{PV}$, anterior parietal cortex, and other cortical areas as well. White lines mark architectonic boundaries. Other conventions as in previous figures. relative to S-I and S-II in squirrels (Krubitzer et al., 1986). In both squirrels and marmosets, PV has a very similar somatotopic organization, and in both mammals, $\mathrm{PV}$ receives inputs from S-I (3b) and from S-II. To conclude that PV is homologous in squirrels and marmosets implies, of course, that PV is a somatosensory area that exists in a wide range of mammals.

Presently, evidence for the existence of PV in other mammals, including higher primates, is limited. However, in macaque monkeys, S-II projects to insular fields rostral to S-II (Fig. 20) that are responsive to cutaneous stimuli (Robinson and Burton, 1980; Friedman et al., 1986) and one of these fields could be PV. Neurons in recording sites from cortex just rostral to S-II in owl monkeys were responsive to cutaneous stimuli, and the same region receives a projection from area $3 \mathrm{~b}$ (Cusick et al., 1989). In addition to squirrels, there is anatomical evidence for PV in rats and mice as well (see Krubitzer et al., 1986, for review). In cats, S-IV (Fig. 20) is in a similar, although slightly ventral, location to PV in squirrels and marmosets, is organized in a similar topographic manner and shares similar patterns of connections as PV in squirrels (Clemo and Stein, 1982, 1983; Burton and Kopf, 1984; see Krubitzer et al., 1986, for review). Taken together, accumulating evidence suggests that PV is a somatosensory area common to all mammals.

\section{$M-I$ and $S M A$}

The connections of area $3 \mathrm{~b}$ and S-II with motor cortex and the SMA were roughly topographic. Injections limited to certain body regions in these 2 representations produced label that had a tendency to be more rostrally located in SMA and more laterally located in M-I after injections in cortex devoted to the face and forelimb than trunk and hindlimb. Both SMA and M-I have somatotopic organizations in which the hindlimb, forelimb, and face are represented in a caudorostral sequence in SMA and a mediolateral sequence in M-I (see Gould et al., 1986). Both representations, however, are more complex than those in area $3 \mathrm{~b}$ and S-II, so that similar body movements can often be elicited from several separate locations in M-I and SMA. Thus, connections with these fields may be less topographic than connections between sensory fields. In addition, sensory information from a given part of the body might usefully relate to movements of several body parts. Thus, heterotopic connections could be functionally significant.

\section{Processing sequences in somatosensory cortex}

Similarities and differences in the organization and connections of somatosensory cortex in rodents, cats, and New World and Old World monkeys (Fig. 20) suggest that a basic processing sequence present in many mammals has been modified in New World monkeys, and even more so in Old World monkeys (Fig. 21). In rodents, S-I, S-II, and PV appear to represent successive early stages in processing (Krubitzer et al., 1986; Krubitzer and Kaas, 1987), with later stages in parietal rhinal cortex (PR) possibly leading to limbic structures that are critical for tactile recognition and memory (see Mishkin, 1979; Friedman et al., 1986). Some of the processing is serial, depending on a relay from the ventroposterior nucleus (VP) to S-I, from S-I to S-II, and from S-II to PV. However, earlier stations in this sequence also directly access later stations. Thus, VP, at least in squirrels (Krubitzer and Kaas, 1987), also projects to S-II, and S-I projects to PV as well as S-II. In addition, S-I, S-II, and PV all project 


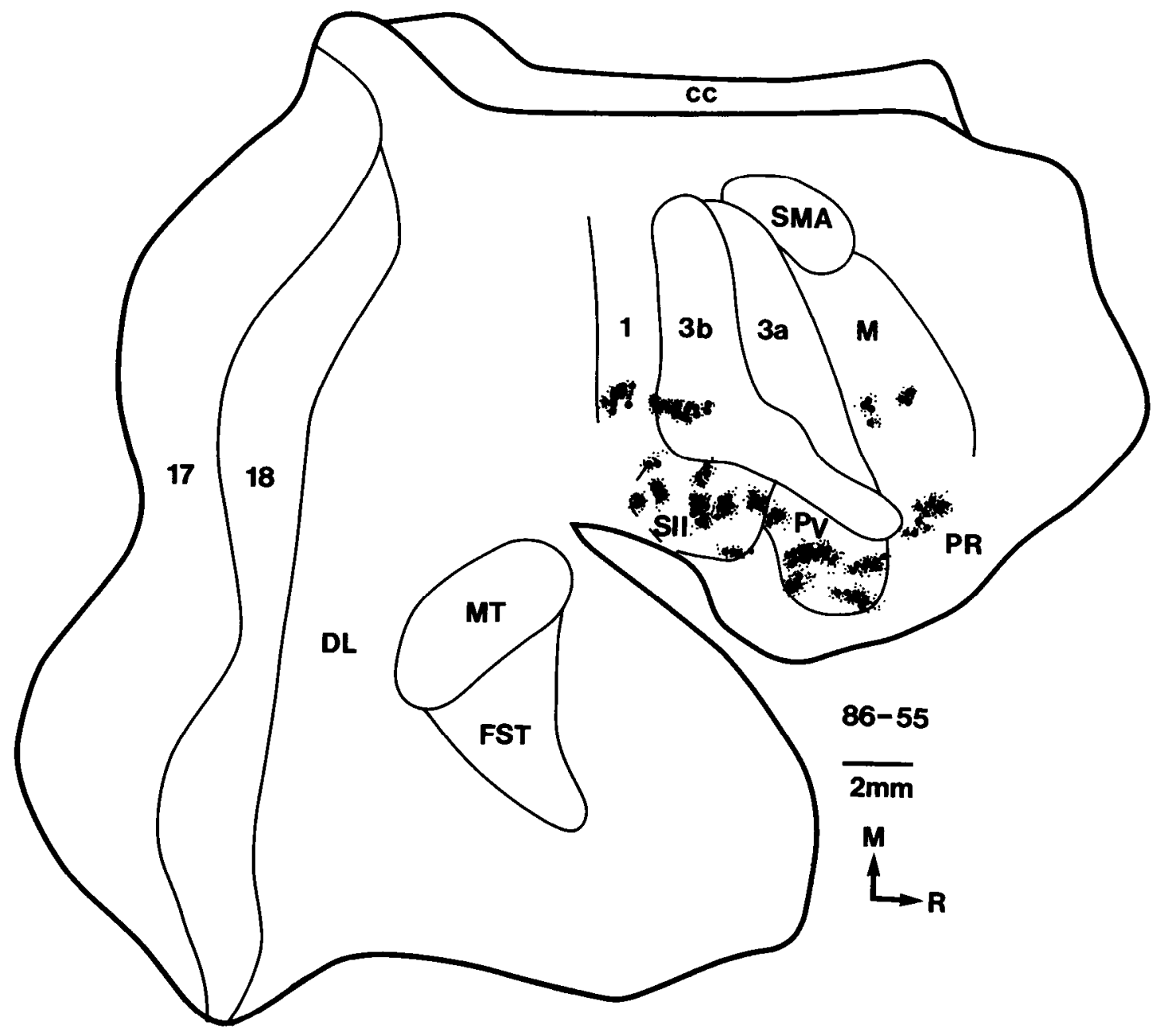

Figure 19. Distribution of callosally transported tracer after an injection of WGA-HRP into the hand representation of S-II in the contralateral hemisphere in marmoset 86-55 (see Fig. 18). The injection in S-II in the opposite hemisphere resulted in dense label in S-II and PV and sparser amounts of label in $3 \mathrm{~b}, 1$, and motor cortex.

to PR. While serial processing may be very important, the more direct paths can bypass early stages. For example, information processed in S-I has the potential of bypassing further processing in S-II and PV by directly relaying to further processing stations in PR. The importance of these parallel connections is clear in that for many mammals, S-II does not depend on S-I for activation (see Garraghty et al., 1990).

In marmosets, this basic processing framework has been modified. The dense projections from S-I (3b) to area 1 suggest that cortex caudal to S-I ( $3 b$ ) has been elaborated and become more significant for somatosensory processing. Area 1 also projects to S-II. In addition, S-II has become dependent on S-I (3b) for direct information about cutaneous receptors (Garraghty et al., 1990) and receives little input from the ventroposterior nucleus (Krubitzer and Kaas, 1987). Thus, middle stages have been added or enhanced, and cutaneous activation of S-II has become more dependent on input from S-I (3b) (Pons et al., 1987, 1988; Garraghty et al., 1990).

New World monkeys of the Cebidae family are more specialized in that area 1 , which is poorly activated by cutaneous stimuli in marmosets (Carlson et al., 1986), is very responsive to light tactile stimuli in owl monkeys (Merzenich et al., 1978), squirrel monkeys (Sur et al., 1982; Cusick et al., 1985), and cebus monkeys (Felleman et al., 1983). Thus, the significance of area 1 as a processing station for cutaneous information in the Cebidae radiation of New World monkeys has been further increased. Finally, there is some activation by cutaneous stimuli of neurons in parts of area 2 in squirrel monkeys and cebus monkeys. Thus, connections from areas $3 b$ and 1 have enhanced the role of cortex caudal to area 1 in the processing of cutaneous information (see Kaas and Pons, 1988, for review).

In Old World monkeys, information is available only for macaque monkeys, but here further elaborations of somatosensory cortical processing stations arc apparent. The ventroposterior nucleus not only projects to $S-I$ (3b) and area 1 , but also to the part of area 2 representing the hand (Pons and Kaas, 1985). Areas $3 b, 1$, and much of area 2 are highly responsive to cutaneous stimuli (Kaas et al., 1979; Nelson et al., 1980; Killackey et al., 1983; Pons and Kaas, 1986), and all of these fields project to S-II (Pons and Kaas, 1986, for review). In addition, parts of area 5 caudal to area 2 receive inputs from areas 1 and 2 , and area 5 in turn projects to S-II (Friedman et al., 1986; Pons and Kaas, 1986). Thus, these middle level stages, including area 1, area 2, and parts of area 5 are even more elaborated in the Old World macaques and have become very important in processing of cutaneous information. They operate 


\section{MARMOSET}
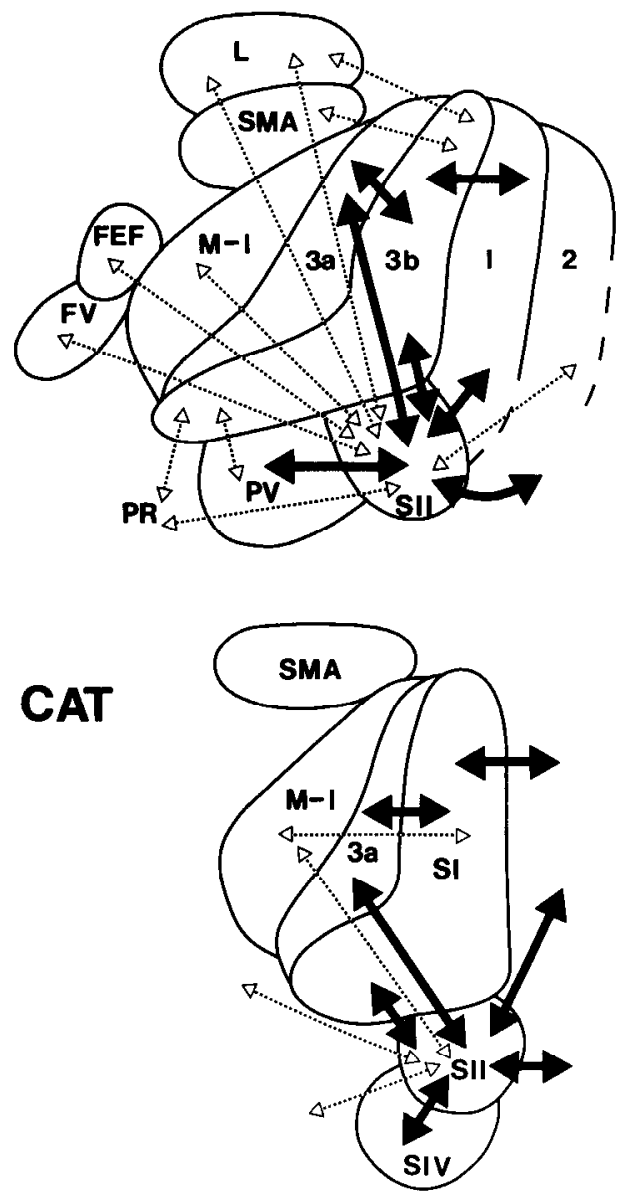

MACAQUE

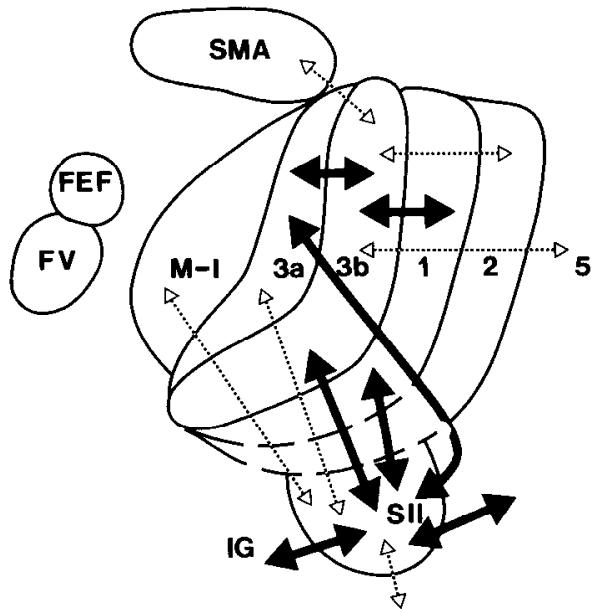

SQUIRREL

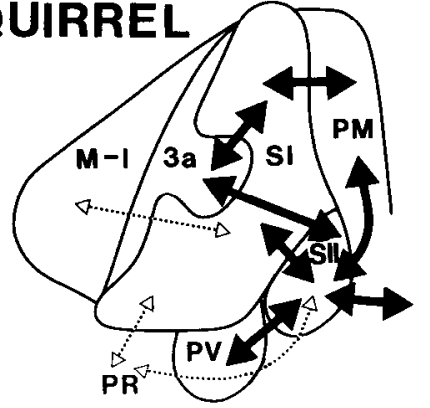

Figure 20. Ipsilateral cortical connections of area $3 b$ or S-I in mammals. Large arrows indicate connections found in all 4 groups (see text for references). In macaques, S-II is commonly shown as bordering area 2 , but the common border of S-II with $3 b$ or $S-I$ in all other mammals suggests that this relationship may occur in macaques as well. Areas $3 a, 3 b, 1$, and 2 are architectonic and functional subdivisions of anterior parictal cortex. $M I$, primary motor cortex; $S M A$, supplementary motor area; $L$, limbic cortex of the medial wall; $F E F$, frontal eye field; $F V$, frontal visual area; $S-I I$, second somatosensory area; $P V$, parietal rhinal area; $P R$, rostroventral parietal cortex; $P M$, parietal medial area. partly in a serial manner and partly in parallel with all fields projecting to S-II.

\section{$S-I I$ connections with visuomotor fields of the frontal lobe}

The present results provide evidence that S-II is interconnected with at least 2 and possibly 3 visuomotor fields of the frontal lobe (Fig. 20). In other studies on marmosets (Kaas and Krubitzcr, 1988), we have defined the classical frontal eye field, FEF, by microstimulation procedures and have related the field to an oval of moderate myelination in brain sections cut parallel to the surface of cortex. The FEF is densely interconnected with the frontal visual area, FV (Huerta et al., 1987), and FV also

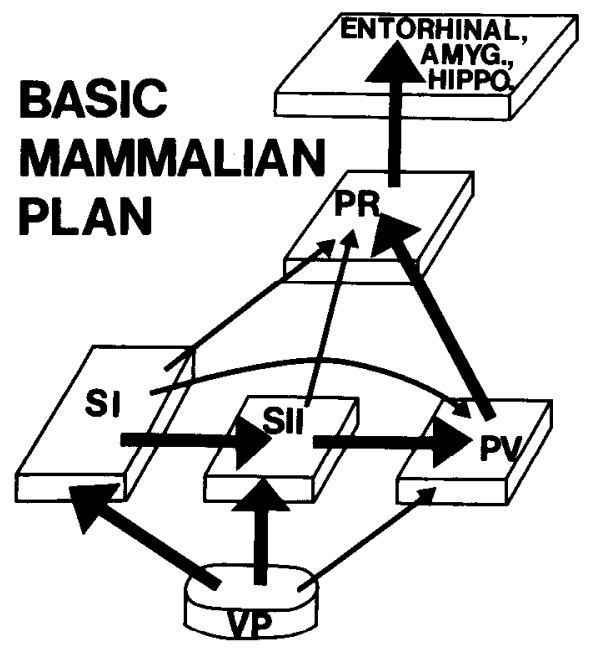

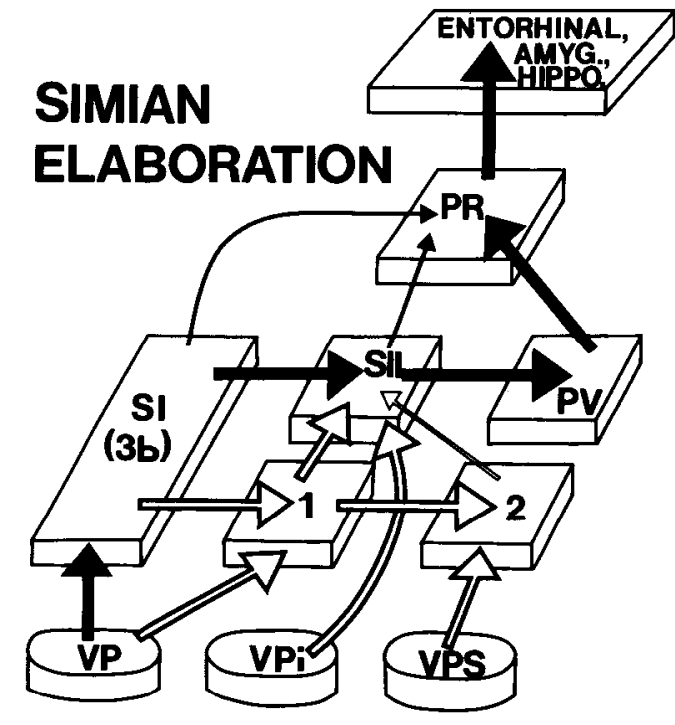

Figure 21. Summary of hierarchical and parallel connections of somatosensory thalamus and cortex schematized for mammals in general (left) and simians (right). Solid thick black arrows denote hierarchical processing sequences in the general mammalian plan and in simians; solid thin arrows show other parallel connections. For simians, the basic mammalian processing sequence is retained. However, an elaboration of an additional processing sequence (open large arrows) is apparent as well. 
has interconnections with several visual areas of the temporal lobe (Kaas and Krubitzer, 1988). In marmosets and other monkeys (Kaas and Krubitzer, 1988), the middle temporal visual area, MT, projects largely to FV and very little to FEF. A third visuomotor area in the rostral portion of SMA is sometimes referred to as the supplementary eye field, SEF (Gould et al., 1986; Huerta et al., 1987; Schlag and Schlag-Rey, 1987). Like the FEF, electrical stimulation of SEF elicits eye movements (Gould et al., 1986; Huerta et al., 1987; Huerta and Kaas, 1988). The SEF is densely interconnected with the FEF (Huerta et al., 1987; Huerta and Kaas, 1988). In the present investigation injections in S-II produced label in FEF, FV, and rostral parts of SMA that could include SEF. Thus, higher stations in the cortical processing of tactile information potentially influence frontal lobe centers involved in the initiation and control of eye movements. Perhaps neurons activated in S-II signal cortical visuomotor centers that gaze should be directed toward some part of the body surface.

\section{Callosal connections of $3 b$ and $S-I I$}

Callosal connections were investigated with multiple injections in area $3 \mathrm{~b}$ and single injections in S-II. While the callosal connections of "S-I" (3a, 3b, 1, and 2) in monkeys have been described (e.g., Pandya and Vignolo, 1968, 1971; Jones and Powcll, 1969b; Boyd et al., 1971; Jones et al., 1975; Shanks et al., 1975, 1985; Jones and Hendry, 1980; Caminiti and Sbriccoli, 1985; Conti et al., 1986; Manzoni et al., 1986), callosal connections specific to area $3 \mathrm{~b}$ (S-I proper) have not been reported for any primate. In marmosets, area $3 \mathrm{~b}$ has callosal connections with areas $3 \mathrm{~b}, \mathrm{~S}-\mathrm{II}$, and PV. Similar connections may exist in macaque monkeys, since injections of HRP involving both areas 1 and 3 b demonstrate interconnections with homotopic regions of contralateral areas 1 and $3 b$ (Conti et al., 1986) and S-II (Manzoni et al., 1986). In these reports, some connections outside of S-II, located in insular granular cortex, IG, could be in PV. In cats (Jones and Powell, 1968; Caminiti et al., 1979), tree shrews (Weller et al., 1987), and squirrels (Krubitzer et al., 1986), S-I connects with S-I and S-II of the opposite hemisphere, and in squirrels, at least, $\mathrm{S}$-II also interconnects callosally with PV, as well as motor cortex.

Even though the strip injection in $3 \mathrm{~b}$ in marmosets involved considerable portions of area $3 b$, the callosal label in areas $3 b$, $\mathrm{S}-\mathrm{II}$, and PV was extremely restricted. In $3 \mathrm{~b}$, the callosal label was concentrated just medial and just lateral to cortex representing the hand, in agreement with the considerable evidence that cortex in $3 \mathrm{~b}$ devoted to the hand, is nearly devoid of callosal connections (e.g., Jones and Hendry, 1980; Killackey et al., 1983). The dense label lateral to the hand representation is in the position of the myelin sparse insert in area $3 \mathrm{~b}$ (Fig. $8, A, B$, $D)$ that separates the hand and face regions. The concentration of callosal connections in architectonically distinct zones is reminiscent of the patterns in rats, where the myelin-poor dysgranular cortex has dense callosal connections, while the myelindense granular cortex is almost free of callosal connections (Akers and Killackey, 1978; Olivarria et al., 1984). Thus, in primates, $3 \mathrm{~b}$ may contain modules created by or related to separate sources of afferent inputs.

Our injections in S-II produced contralateral foci of label in both S-II and PV. However, detectable levels of label did not occur in area $3 \mathrm{~b}$, even though the injections in area $3 \mathrm{~b}$ demonstrated the existence of such connections. The absence of transported tracer in contralateral area $3 \mathrm{~b}$ could be a result of our centering the injections in cortex representing the forepaw, since the homotopic portion of $3 \mathrm{~b}$ has few if any callosal connections. In macaque monkeys, Manzoni and colleagues (1986) found that an injection in the part of S-II related to the hand labeled many neurons in contralateral S-II but few neurons in contralateral "S-I." S-II connections with S-II of the opposite hemisphere have also been reported for squirrels (Krubitzer et al., 1986), tree shrews (Weller et al., 1987), and cats (Caminiti et al., 1979; Barbaresi et al., 1989).

\section{Appendix}

\section{List of abbreviations of cortical fields in marmosets}

A-I, primary auditory area; FEF, frontal eye field; FST, fundal superior temporal area; FV, frontal visual area; L, limbic cortex; M-I, primary motor area; MT, middle temporal visual area; PR, rostroventral parietal cortex; PV, parietal ventral area; S-I, primary somatosensory area; S-II, second somatosensory area; R, rostral auditory area; SEF, supplementary eye field; SMA, supplementary motor area; V-I, primary visual area; and V-II, second visual area.

\section{References}

Akers, R. M., and H. P. Killackey (1978) Organization of corticocortical connections in the parietal cortex of the rat. J. Comp. Neurol. 181: $513-538$.

Alloway, K. D., and H. R. Burton (1985) Homotypical ipsilateral cortical projections between somatosensory areas I and II in the cat. Neuroscience 14: 15-35.

Barbaresi, P., S. Bernardi, and T. Manzoni (1989) Callosal connections of SII and SIV. J. Comp. Neurol. 283: 355-373.

Beattie, J. (1927) The anatomy of the common marmoset (Hapale jacchus). J. Proc. Zool. Soc. London 1927: 543-718.

Boyd, E. H., D. N. Pandya, and K. E. Bignall (1971) Homotopic and nonhomotopic interhemispheric cortical projections in the squirrel monkey. Exp. Neurol. 32: 256-274.

Burton, H. (1986) Second somatosensory cortex and related areas. In Cerebral Cortex, Vol. 5, E. G. Jones and A. Peters, eds., pp. 31-98, Plenum, New York.

Burton, H., and M. Carlson (1986) Second somatic sensory cortical area (SII) in a prosimian primate, Galago crassicaudatus. J. Comp. Neurol. 247: 200-220.

Burton, H., and E. M. Kopf (1984) Ipsilateral cortical connections from the second and fourth somatic sensory areas in the cat. J. Comp. Neurol. 225: 527-553.

Burton, H., G. Mitchell, and D. Brent (1982) Second somatic sensory area in the cerebral cortex of cats: Somatotopic organization and cytoarchitecture. J. Comp. Neurol. 210: 109-135.

Caminiti, R., and A. Sbriccoli (1985) The callosal system of the superior parietal lobule in the monkey. J. Comp. Neurol. 237: 85-99.

Caminiti, R., G. M. Innocenti, and T. Manzoni (1979) The anatomical substrate of callosal messages from SI and SII in the cat. Brain Res. 35: $295-314$

Carlson, M., M. F. Huerta, C. G. Cusick, and J. H. Kaas (1986) Studies on the evolution of multiple somatosensory representations in primates: The organization of anterior parietal cortex in the New World callitrichid, Saguinus. J. Comp. Neurol. 246: 409-426.

Carvell, G. E., and D. J. Simons (1986) Somatotopic organization of the second somatosensory area (SII) in the cerebral cortex of the mouse. Somatosensory Res. 3: 213-237.

Clemo, H. R., and B. E. Stein (1982) Somatosensory cortex: A 'new' somatotopic representation. Brain Res. 235: 162-168.

Clemo, H. R., and B. E. Stein (1983) Organization of a fourth somatosensory area of cortex in cat. J. Neurophysiol. 50: 910-925.

Conti, F., M. Fabri, and T. Manzoni (1986) Bilateral receptive fields and callosal connectivity of the body midline representation in the first somatosensory area of primates. Somatosensory Res. 3: 273289.

Cusick, C. G., and M. M. Manning (1988) Somatotopic organization and thalamic connections of the second somatosensory area, S-II, in squirrel monkeys. Soc. Neurosci. Abstr. 14: 222. 
Cusick, C. G., D. A. Steindler, and J. H. Kaas (1985) Corticocortical and collateral thalamocortical connections of postcentral somatosensory cortical areas in squirrel monkeys: A double-labeling study with radiolabeled wheatgerm agglutinin and wheatgerm agglutinin conjugated to horseradish peroxidase. Somatosensory Res. 3: 1-31.

Cusick, C. G., J. T. Wall, D. J. Felleman, and J. H. Kaas (1989) Somatotopic organization of the lateral sulcus of owl monkeys: Area $3 b$, S-II, and a ventral somatosensory area. J. Comp. Neurol. 282. $169-190$.

Felleman, D. J., R. J. Nelson, M. Sur, and J. H. Kaas (1983) Representations of the body surface in areas $3 \mathrm{~b}$ and 1 of postcentral parietal cortex of cebus monkeys. Brain Res. 268: 15-26.

Friedman, D. P. (1983) Laminar patterns of termination of corticocortical afferents in the somatosensory system. Brain Res. 273: 147151.

Friedman, D. P., E. A. Murray, J. B. O'Neill, and M. Mishkin (1986) Cortical connections of the somatosensory fields of the lateral sulcus of Macaques: Evidence for a corticolimbic pathway for touch. J. Comp. Neurol. 252: 323-347.

Gallayas, F. (1979) Silver staining of myelin by means of physical development. Neurology 1: 203-209.

Garraghty, P. E., T. P. Pons, and J. H. Kaas (1988) SII in New World monkeys depends upon inputs from SI for its activation. Soc. Neurosci. Abstr. 14: 223 .

Garraghty, P. E., T. P. Pons, and J. H. Kaas (1989) Ablations of areas $3 \mathrm{~b}$ (S-I proper) and $3 \mathrm{a}$ of somatosensory cortex in marmosets deactivate the second and parietal ventral somatosensory areas. Somatosens. Motor Res. (in press).

Gould, H. J., III, C. G. Cusick, T. P. Pons, and J. H. Kaas (1986) The relationship of corpus callosum connections to electrical stimulation maps of motor, supplementary motor, and the frontal eye fields in Owl Monkeys. J. Comp. Neurol. 247: 297-325.

Haight, J. R. (1972) The general organization of somatotopic projections to SII cerebral neocortex in the cat. Brain Res. 44: 483-502.

Herron, P. (1978) Somatotopic organization of mechanosensory projections to SII cerebral neocortex in the raccoon (Procyon iotor). J. Comp. Neurol. 181: 717-728.

Hershkovitz, P. (1977) Living New World Monkeys, Vol. 1. University of Chicago Press, Chicago.

Huerta, M. F., and J. H. Kaas (1988) Connections of the physiologically defined supplementary eye field. Soc. Neurosci. Abstr. 14:159.

Huerta, M. F., J. T. Wall, and J. H. Kaas (1986) Changes in topography of somatosensory cortex after nerve loss in adult and neonatal marmoset monkeys. Soc. Neurosci. Abstr. 12: 954.

Huerta, M. F., L. A. Krubitzer, and J. H. Kaas (1987) Frontal eye field as defined by intracortical microstimulation in squirrel monkeys, owl monkeys, and macaque monkeys. II. Cortical connections. J. Comp. Neurol. 265: 332-361.

Jones, E. G., and S. H. C. Hendry (1980) Distribution of callosal fibers around the hand representations in monkey somatic sensory cortex. Neurosci. Lett. 19: 167-172.

Jones, E. G., and T. P. S. Powell (1968) The ipsilateral cortical connexions of the somatic sensory areas in the cat. Brain Res. 9: 71-94.

Jones, E. G., and T. P. S. Powell (1969a) Connexions of the somatic sensory cortex of the rhesus monkey. I. Ipsilateral cortical connexions. Brain 92: 477-502.

Jones, E. G., and T. P. S. Powell (1969b) Connexions of the somatic sensory cortex of the rhesus monkey. II. Contralateral cortical connexions. Brain 92: 717-730.

Jones, E. G., and S. P. Wise (1977) Size, laminar and columnar distribution of efferent cells in the sensory-motor cortex of monkeys. J. Comp. Neurol. 175: 391-438.

Jones, E. G., H. Burton, and R. Porter (1975) Commissural and cortico-cortical "columns" in the somatic sensory cortex of primates. Science 190: 572-574.

Jones, E. G., J. D. Coulter, and S. H. C. Hendry (1978) Intracortical connectivity of architectonic fields in the somatic sensory, motor and parietal cortex of monkeys. J. Comp. Neurol. 181: 291-348.

Kaas, J. H. (1983) What, if anything, is SI? Organization of first somatosensory area of cortex. Physiol. Rev. 63: 206-231.

Kaas, J. H. (1987a) The organization of neocortex in mammals: Implications for theories of brain function. Annu. Rev. Psychol. 38: 124-151.

Kaas, J. H. (1987b) The organization and evolution of neocortex. In Higher Brain Function, S. P. Wise, ed., pp. 347-378, Wiley, New York.
Kaas, J. H. (1988) Changing concepts of visual cortex organization in primates. In Neuropsychology of Visual Perception, J. W. Brown, ed., pp. 1-32, Lawrence Erlbaum, Hillsdale, NJ.

Kaas, J. H. (1989) The evolution of complex sensory systems in mammals. J. Exp. Biol. 146: 165-176.

Kaas, J. H., and L. A. Krubitzer (1988) Subdivisions of visuomotor and visual cortex in the frontal lobe of primates: The frontal eye field and the target of the middle temporal area. Soc. Neurosci. Abstr. 14: 820.

Kaas, J. H., and T. P. Pons (1988) The somatosensory system of primates. In Comparative Primate Biology, Vol. 4, H. P. Steklis, ed., pp. 421-468, Liss, New York.

Kaas, J. H., R. J. Nelson, M. Sur, C. S. Lin, and M. M. Merzenich (1979) Multiple representations of the body within the primary somatosensory cortex of primates. Science 204: 521-523.

Killackey, H. P., H. J. Gould, III, C. G. Cusick, T. P. Pons, and J. H. Kaas (1983) The relation of corpus callosum connections to architectonic fields and body surface maps in sensorimotor cortex of new and old world monkcys. J. Comp. Neurol. 219: 369-419.

Krubitzer, L. A., and J. H. Kaas (1986) The second somatosensory area in primates: Somatotopic organization, architecture, and connections in marmosets (Callithrix jacchus). Soc. Neurosci. Abstr. 12: 798.

Krubitzer, L. A., and J. H. Kaas (1987) Thalamic connections of three representations of the body surface in somatosensory cortex of gray squirrels. J. Comp. Neurol. 265: 549-580.

Krubitzer, L. A., and J. H. Kaas (1988) Responsiveness and somatotopic organization of anterior parietal field $3 \mathrm{~b}$ and adjoining cortex in newborn and infant monkeys. Somatosens. Motor Res. 6: 179205.

Krubitzer, L. A., M. A. Sesma, and J. H. Kaas (1986) Microelectrode maps, myeloarchitecture, and cortical connections of three somatotopically organized representations of the body surface in the parietal cortex of squirrels. J. Comp. Neurol. 250: 403-430.

Lende, R. A., and C. N. Woolsey (1956) Sensory and motor localization in cerebral cortex of porcupine. J. Neurophysiol. 19:544-563.

Lucier, G. E., D. C. Ruegg, and M. Wiesendanger (1975) Responses of neurones in motor cortex and in area $3 \mathrm{~A}$ to controlled stretches of forelimb muscles in cebus monkeys. J. Physiol. (Lond.) 251: 833853.

Luethke, L. E., L. A. Krubitzer, and J. H. Kaas (1989) Connections of primary auditory cortex in the new world monkey, Saguinus. J. Comp. Neurol. 285: 487-513.

Maendly, R., D. G. Ruegg, M. Wiesendanger, R. Wiesendanger, J. Lagowska, and B. Hess (1981) Thalamic relay for group I muscle afferents of forelimb nerves in the monkey. J. Neurophysiol. 5: $901-$ 917.

Manzoni, T., R. Caminiti, G. Spidalieri, and E. Morelli (1979) Anatomical and functional aspects of the associative projection from somatic area SI to SII. Exp. Brain Res. 34: 453-470.

Manzoni, T., F. Conti, and M. Fabri (1986) Callosal projections from area SII to SI in monkeys: Anatomical organization and comparison with association projections. J. Comp. Neurol. 252: 245-263.

Merzenich, M. M., J. H. Kaas, M. Sur, and C. S. Lin (1978) Double representation of the body surface within cytoarchitectonic areas $3 b$ and 1 in "SI" in the owl monkey (Aotus trivirgatus). J. Comp. Neurol. 181: 41-74.

Mesulam, M. M. (1978) Tetramethylbenzidine for horseradish peroxidase neurohistochemistry: A non-carcinogenic blue reaction product with superior sensitivity for visualizing neural afferents and efferents. J. Histochem. Cytochem. 26: 160-177.

Mishkin, M. (1979) Analogous neural models for tactual and visual learning. Neuropsychologia 17: 139-151.

Nelson, R. J., M. Sur, and J. H. Kaas (1979) The organization of the second somatosensory area (SmII) of the grey squirrel. J. Comp. Neurol. 184: 473-490.

Nelson, R. J., M. Sur, D. J. Felleman, and J. H. Kaas (1980) Representations of the body surface in postcentral parietal cortex of Macaca fascicularis. J. Comp. Neurol. 192: 611-643.

Olivarria, J., R. C. Van Sluyters, and H. P. Killackey (1984) Evidence for the complimentary organization of callosal and thalamic connections within rat somatosensory cortex. Brain Res. 291: 364-368.

Pandya, D. N., and L. A. Vignolo (1968) Interhemispheric neocortical projections of somatosensory areas I and II in the rhesus monkey. Brain Res. 7: 300-303.

Pandya, D. N., and L. A. Vignolo (1971) Intra- and interhemispheric 
projections of the precentral, premotor and arcuate areas in the rhesus monkey. Brain Res. 26: 217-233.

Phillips, C. G., T. P. S. Powell, and M. Wiesendanger (1971) Projection from low-threshold muscle afferents of hand and forearm to area 3a of baboon's cortex. J. Physiol. (Lond.) 217: 419-450.

Pimentel-Souza, F., R. M. Cosenza, G. B. Campos, and J. I. Johnson (1980) Somatic sensory cortical regions of the agouti, Dasyprocta aguti. Brain Behav. Evol. 17: 218-240.

Pons, T. P., and J. H. Kaas (1985) Connections of area 2 of somatosensory cortex with the anterior pulvinar and subdivisions of the ventroposterior complex in macaque monkeys. J. Comp. Neurol. 240: 16-36.

Pons, T. P., and J. H. Kaas (1986) Corticocortical connections of area 2 of somatosensory cortex in macaque monkeys: A correlative anatomical and electrophysiological study. J. Comp. Neurol. 248: 313335.

Pons, T. P., P. E. Garraghty, C. G. Cusick, and J. H. Kaas (1985a) A sequential representation of the occiput, arm, forearm and hand across the rostrocaudal dimension of areas 1,2 , and 5 in macaque monkeys. Brain Res. 335: 350-353.

Pons, T. P., P. E. Garraghty, C. G. Cusick, and J. H. Kaas (1985b) The somatotopic organization of area 2 in macaque monkeys. J. Comp. Neurol. 241: 445-466.

Pons, T. P., P. E. Garraghty, D. P. Friedman, and M. Mishkin (1987) Physiological evidence for serial processing in somatosensory cortex. Science 237: 417-420.

Pons, T. P., P. E. Garraghty, and M. Mishkin (1988) Lesion-induced plasticity in the second somatosensory cortex of adult macaques. Proc. Natl. Acad. Sci. USA 85: 5279-5281.

Pubols, B. H., Jr. (1977) The second somatic sensory area (SmII) of opossum neocortex. J. Comp. Neurol. 174: 71-78.

Robinson, C. J., and H. Burton (1980) Somatotopographic organization in the second somatosensory area of $M$. fascicularis. J. Comp. Neurol. 192: 043-067.

Schlag, J., and M. Schlag-Rey (1987) Evidence for a supplementary eye field. J. Neurophysiol. 57: 179-200.

Shanks, M. F., A. J. Rockel, and T. P. S. Powell (1975) The com- missural fibre connections of the primary somatic sensory cortex. Brain Res. 98: 166-171.

Shanks, M. F., R. C. A. Pearson, and T. P. S. Powell (1985) The callosal connexions of the primary somatic sensory cortex in the monkey. Brain Res. Rev. 9: 43-65.

Sur, M., R. E. Weller, and J. H. Kaas (1981) The organization of somatosensory area II in tree shrews. J. Comp. Neurol. 201: 121133.

Sur, M., R. J. Nelson, and J. H. Kaas (1982) Representations of the body surface in cortical areas $3 \mathrm{~b}$ and 1 of squirrel monkeys: Comparisons with other primates. J. Comp. Neurol. 211: 177-192.

Van Essen, D. C., and J. H. R. Maunsell (1983) Hierarchical organization and functional streams in the visual cortex. TINS $6: 370$ 375

Vogt, B. A., and D. N. Pandya (1978) Cortico-cortical connections of somatic sensory cortex (areas 3, 1 and 2 ) in the rhesus monkey. $J$. Comp. Neurol. 177: 179-192.

Wall, J. T., M. F. Huerta, and J. H. Kaas (1986) Somatosensory cortex topography after nerve repair in neonatal marmoset monkeys. Soc. Neurosci. Abstr. 12: 1436.

Welker, C., and M. M. Sinha (1972) Somatotopic organization of SmII cerebral neocortex in albino rat. Brain Res. 37: 132-136.

Welker, E., P. V. Hoogland, and H. Van der Loos (1988) Organization of feedback and feedforward projections of the barrel cortex: A PHA-L study in the mouse. Exp. Brain Res. 73: 411-435.

Weller, R. E., M. Sur, and J. H. Kaas (1987) Callosal and ipsilateral cortical connections of the body surface representations in SI and SII of tree shrews. Somatosens. Res. 5: 107-133.

White, P. F., W. L. Way, and A. J. Trevor (1982) Ketamine-Its pharmacology and therapeutic uses. Anesthesiology 56: 119-136.

Whitsell, B. L., L. M. Petrucelli, and G. Werner (1969) Symmetry and connectivity in the map of the body surface in somatosensory area II of primates. J. Neurophysiol. 32: 170-183.

Wiesendanger, M., and T.S. Miles (1982) Ascending pathway of lowthreshold muscle afferents to the cerebral cortex and its possible role in motor control. Physiol. Rev. 62: 1234-1270. 